\title{
Collection of the Abstracts of the 2019Sp PMD: Translational Myology and Mobility Medicine
}

\author{
Ugo Carraro $(1,2)$
}

(1) Interdepartmental Research Centre of Myology (CIR-Myo), Department of Biomedical Sciences, University of Padova, Italy; (2) A\&C M-C Foundation for Translational Myology, Padova, Italy

This article is distributed under the terms of the Creative Commons Attribution Noncommercial License (CC BY-NC 4.0) which permits any noncommercial use, distribution, and reproduction in any medium, provided the original author(s) and source are credited.

\begin{abstract}
The Interdepartmental Research Centre of Myology (CIR-Myo), Department of Biomedical Sciences, University of Padova, Italy and the A\&C M-C Foundation for Translational Myology, Padova, Italy organized with the scientific support of Helmut Kern, Jonathan C. Jarvis, Viviana Moresi, Marco Narici, Feliciano Protasi, Marco Sandri and Ugo Carraro, the 2019SpringPaduaMuscleDays: Translational Myology and Mobility Medicine, an International Conference held March 28-30, 2019 in Euganei Hills and Padova (Italy). Presentations and discussions of the Three Physiology Lectures and of the seven Sessions (I: Spinal Cord Neuromodulation and h-bFES in SC; II: Muscle epigenetics in aging and myopathies; III: Experimental approaches in animal models; IV: Face and Voice Rejuvenation; V: Muscle Imaging; VI: Official Meeting of the EU Center of Active Aging; VII: Early Rehabilitation after knee and hip replacement) were at very high levels. This was true in the past and will be true in future events thanks to researchers and clinicians who were and are eager to attend the PaduaMuscleDays.
\end{abstract}

Key Words: Abstracts, PaduaMuscleDays, Translational Myology and Mobility Medicine

Eur J Transl Myol 29 (1): 46-78, 2019

\section{Abstracts of the 2019Spring Padua Muscle Day, March 28-30}

\section{March 28, 2019 - Hotel Augustus, Euganei Hills (Padova), Italy}

\section{SESSION I: Spinal Cord Neuromodulation and h-bFES in SCI}

Epidural Stimulation for the restoration of function following motor complete spinal cord injury

Claudia A. Angeli

Spinal Cord Injury Research Center, Louisville, KY, USA

E-mail: ClaudiaAngeli@kentuckyonehealth.org
We have previously shown that chronic, motor complete SCI individuals can progressively recover voluntary and standing ability when lumbosacral spinal cord epidural stimulation (scES) is applied with task- and individualspecific parameter. ${ }^{1-4}$ The aim of this study was to investigate the effects of two different activity-based training paradigms with scES on standing and stepping ability in individuals with chronic complete SCI using scES. Eight individuals with chronic, motor complete spinal cord injury (SCI) were implanted with a spinal cord epidural stimulation unit. All research participants received an implant of a 16-electrode array on the dura encompassing L1-S1 cord segments. Four individuals performed approximately 80 sessions of stand training with scES ( 5 days/week; 1 hour per session) followed by 80 sessions of step training with scES (5 days/week; 1 hour per session) (Group 1). Four other individuals 


\section{Sp PMD Collection of the Abstracts}

Eur J Transl Myol 29 (1):46-78 2019

(Group 2) performed an interleaving stand-step training with task-specific scES, which consisted of stand training and step training that alternated every session until for a total of approximately 160 sessions 5 days/week; 1 or 2 hour per day). The interleaving stand-step training with scES promoted significant recovery of standing and stepping ability in all four chronic complete SCI individuals, and seemed more effective than the previous paradigm in which stand training was completed prior to step training. 5 This indicates that the human spinal circuitry can learn to generate motor patterns effective for standing and stepping while retraining both tasks with task-specific scES. For complex tasks such as stepping and walking, the human lumbosacral circuitry was transformed into functional states that generated independent steps when optimized epidural stimulation was present and task specific proprioception for stepping was ongoing. However, independent stepping only occurred when the individual was driving specific intent for walking. This unexpected finding showed that de novo functional supraspinal connections had emerged to reestablish control of aspects of locomotion in individuals who had been clinically diagnosed with motor complete spinal cord injury. All eight individuals were also able to continue standing and in one case walking in the home and community in the presence of task-specific epidural stimulation. The spinal circuitry has potential to drive recovery after severe spinal cord injuries if provided with the appropriate retraining in a specific central state of excitability.

1. S. Harkema et al., Effect of epidural stimulation of the lumbosacral spinal cord on voluntary movement, standing, and assisted stepping after motor complete paraplegia: a case study. Lancet 377, 1938-1947 (2011).

2. C. A. Angeli, V. R. Edgerton, Y. P. Gerasimenko, S. J. Harkema, Altering spinal cord excitability enables voluntary movements after chronic complete paralysis in humans. Brain 137, 1394-1409 (2014).

3. E. Rejc, C. Angeli, S. Harkema, Effects of Lumbosacral Spinal Cord Epidural Stimulation for Standing after Chronic Complete Paralysis in Humans. PloS one 10, e0133998 (2015).

4. E. Rejc, C. A. Angeli, N. Bryant, S. J. Harkema, Effects of Stand and Step Training with Epidural Stimulation on Motor Function for Standing in Chronic Complete Paraplegics. J Neurotrauma (2016).

5. C. A. Angeli et al., Recovery of Over-Ground Walking after Chronic Motor Complete Spinal Cord Injury. N Engl J Med (2018).

The future of Restorative Neurology in SCI: Driving Translational pipeline from basic research to clinical rehabilitation

\section{Humberto Cerrel-Bazo}

ORAS/ULSS 2 Rehabilitation Hospital, Spinal Cord Injury Unit \& Severe Acquired Brain Injury Unit, Motta di Livenza, Treviso, Italy

\section{E-mail: hcb57@yahoo.com}

The effects of central nervous system (CNS) injury/disease include altered sensation and pain and impaired control of movement, paralysis and spasticity.
Early neurophysiological studies of SCI (1980's) found that in many clinically complete AIS " $A$ " patients there was evidence of transmission of signals across the lesion both caudal and rostral as detected by poly EMG for the motor tracts and evoked potentials for the sensory pathways. ${ }^{1}$ Initially this findings were considered to be artefacts. This dogmatic view was revised when these findings were confirmed by the neuropathology of SCI which demonstrated that many clinically complete cases of SCI had in fact to some degree preserved white matter traversing the lesion as demonstrated by Kakulas. ${ }^{2}$ A meticulous structural and functional criteria for the categorization of spinal cord injuries leads to a complex problem of taxonomy. Therefore, Dimitrijevic et al. ${ }^{3}$ chose to simplify such a complex categorization by estimating the population of functional axons of the long descending tracts through the description of the features of volitional motor control of the motor units below the SCI. From this standpoint, he proposed two categories of spinal cord injury: i) "reduced anatomy," characterized by diminished but organized brain motor control below the level of injury, and ii) "new anatomy," which results from neuro-biologic responses of the central nervous system (CNS) to the injury. Consequently it mat be said that a critical number and type of the survival neurons in the site of injury survive, but apparently are not capable of conducting action potentials, and thus are electrically non-responsive or little responsive. These neuronal networks within the injured region can be neuromodulated to a transformed state of electrical competency. The above mentioned two factors may facilitate the activity-dependent reorganization of neuronal networks in the spinal cord and brain, and propriospinal networks may play a critical role in driving this activity-dependent reorganization after injury. Realtime proprioceptive input to spinal networks provides the template for reorganization of spinal networks that play a leading role in the level of coordination of motor pools required to perform a given functional task. This fertile "new anatomy" territory have allow biological, clinical, neurosurgical, neurophysiological, neuro-engineers \& neurorehab researchers to modify residual, altered, yet remaining nervous system function. This presentation addresses therapeutic paradigm of some of the mechanisms that might explain the effects of neuromodulation and how task oriented training approach may facilitates the recovery of volitional motor control in spinal cord injury subjects as part of the restorative neurology dogma. The good, the bad and the ugly of restorative neurology.

1. Dimitrijevic MR. Residual motor functions in spinal cord injury. In: Waxman SG.(Ed) Functional Recovery in Neurological Disease. New York: Raven Press; 1988, p. 139-155.

2. Kakulas BA, Kaelan C. The neuropathological foundations for the restorative neurology of spinal cord injury. Clin Neurol Neurosurg. 2015;129 Suppl 1:S1-7. doi: 10.1016/j.clineuro.2015.01.012. Epub 2015 Jan 29.

3. Dimitrijevic MR, Persy I, Forstner C, Kern H, Dimitrijevic MM. Motor control in the human spinal cord. Artif Organs 2005;29:216-9. 


\section{Sp PMD Collection of the Abstracts}

Eur J Transl Myol 29 (1):46-78 2019

Two-year h-bFES improves structure and function of muscles in complete thoracic-level SCI

Hofer C,1, Loefler S, 1,2, Ravara B,3,4, Albertin G,5, Zampieri S,2,3, Pond A,6, Cerrel-Bazo H,7, Kern H,2, Carraro $U, 4,6$,

1. Ludwig Boltzmann Institute of Electrical Stimulation and Physical Rehabilitation, Vienna, Austria;

2. Physiko- und Rheumatherapie, St. Poelten, Austria;

3. Department of Biomedical Science,

Interdepartmental Research Centre of Myology, University of Padova, Italy; 4. A\&C M-C Foundation for Translational Myology, 35137 Padova, Italy; 5. Department of Neuroscience, University of Padova, Italy;6. Anatomy Department, Southern Illinois

University School of Medicine, Carbondale, IL, USA; 7. ORAS Rehabilitation Hospital at High Level of Specialization, Motta di Livenza, Treviso, Italy

\section{E-mail: christian.hofer@rehabilitationresearch.eu}

Spinal cord injury (SCI) can result in drastic skeletal muscle atrophy and home-based functional electrical stimulation (h-bFES) is a treatment which has been shown to curtail muscle loss in atrophic situations. ${ }^{1-3} \mathrm{We}$ investigated muscle atrophy progression in thoracic-level paraplegics at various years after SCI and the effect of two years of h-bFES. Force was measured as knee
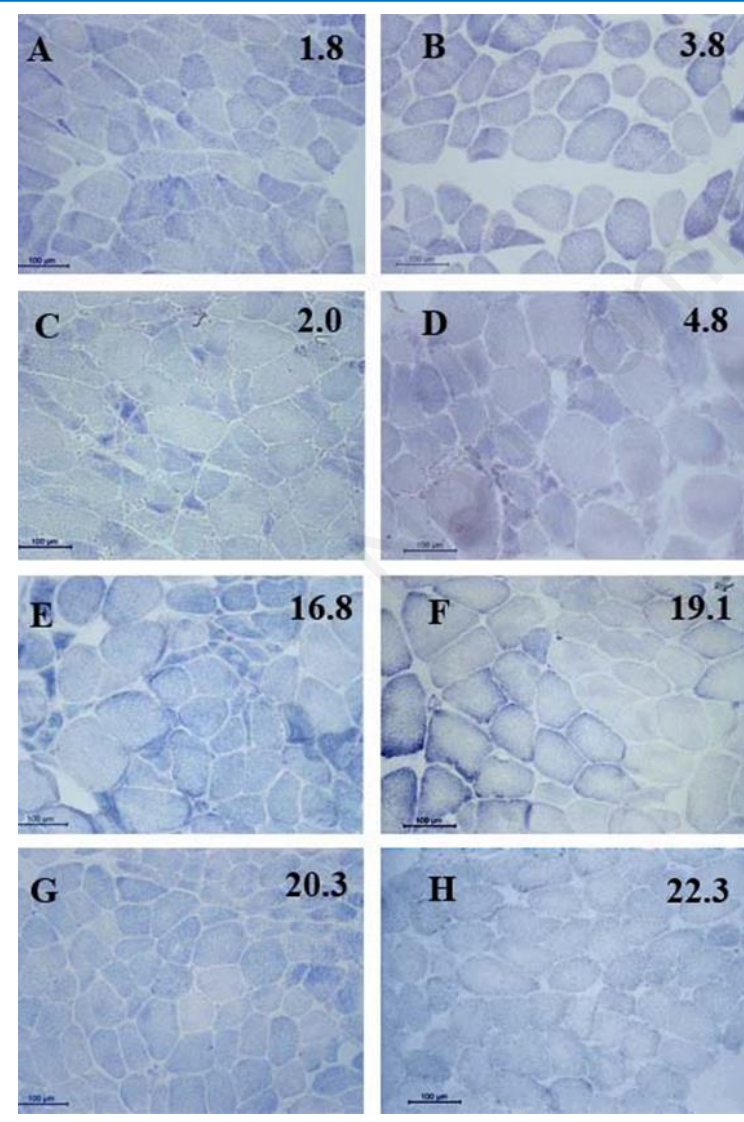

Fig. 1. SDH stain of thoracic-level SCI before (left panels) and after (right panels) two years of hbFES. Numbers in right corners indicate time from spinal cord injury in years. extension torque; macroscopic bulk was determined by color computer tomography; and tissue composition and muscle fiber properties were evaluated by histology and histochemistry. By comparing two groups of persons with thoracic-level SCI (Group 1. Mid-term, 2-4 years post SCI versus Group 2. Long-term, 15-20 years post SCI), we found: 1 . Before h-bFES, force and size of thigh muscles were only slightly decreased in the long-term group when compared to the mid-term SCI persons; 2 . The difference in average size of muscle fibers between the groups was small; 3 . In Group 2 there was a decrease in Succinyl Dehydrogenase, a biomarker of mitochondrial content, which did not increased after 2 years of h-bFES, suggesting that the applied protocol did not significantly affected muscle fiber metabolism, and thus the resistance to fatigue of the stimulated muscle. ${ }^{4}$ On the other hand, 2-year h-bFES-training substantially reversed the atrophy process. Indeed, after 2-years hbFES muscle fibers presented an almost normal sedentary muscle size value; however, histochemical features did not reversed to the same extent (Figure 1). The stable muscle atrophy that characterized long-lasting spastic paraplegia, and the fact that the extent of h-bFES recovery did not correlated with time from SCI, strongly suggest that there are no upper-time limits to begin hbFES in complete thoracic-level paraplegia. Our results show that the hope, related to improvements of spinal cord neuromodulation, may be extended to the entire world population of thoracic-level paraplegic persons.

1. Carraro U, Kern H, Gava P, et al. Biology of Muscle Atrophy and of its Recovery by FES in Aging and Mobility Impairments: Roots and By-Products. Eur J Transl Myol. 2015;25(4):221-30. doi: 10.4081/ejtm.2015.5272. eCollection 2015 Aug 24.

2. Kern H, Hofer C, Loefler S, et al. Atrophy, ultrastructural disorders, severe atrophy and degeneration of denervated human muscle in SCI and Aging. Implications for their recovery by Functional Electrical Stimulation, updated 2017. Neurol Res 2017. doi.org/10.1080/01616412.2017. 1314906.

3. Carraro U, Kern H, Gava P, et al. Recovery from muscle weakness by exercise and FES: lessons from Masters, active or sedentary seniors and SCI patients. Aging Clin Exp Res. 2017;29(4):579-590. doi: 10.1007/s40520-016-0619-1. Epub 2016 Sep 3. Review.

4. Hofer C, Loefler S, Kern H, et al. Two years of FES training improves muscle fibers of thigh muscles in long-term thoracic level-complete spinal cord injury. Biol Eng Med 2018;3(3):1-5. doi: 10.15761/BEM.1000S1002.

$$
* * * * *
$$

The influence of electrode configuration and pulse shape on neural activation

Steffen Eickhoff, Jonathan C Jarvis

Liverpool John Moores University, UK

E-mail: J.C.Jarvis@ljmu.ac.uk 


\section{Sp PMD Collection of the Abstracts}

Eur J Transl Myol 29 (1):46-78 2019

Our experiments on the influence of pulse shaping parameters in rectangular stimulus waveforms have shown that the introduction of an Interphase Gap (IPG) i.e. a delay between the cathodic and anodic stimulation phase, slightly lowers the activation thresholds in monopolar stimulation (in agreement with published literature $)^{1-3}$ but surprisingly, it increases thresholds in bipolar stimulation. We have tested the hypothesis that the middle field transition of a biphasic stimulus, that can under certain conditions have an inhibiting effect in monopolar stimulation, ${ }^{1}$ is the effective transition in bipolar stimulation. Weakening this field transition by introducing an IPG therefore would result in increased thresholds in a bipolar setup. We measured

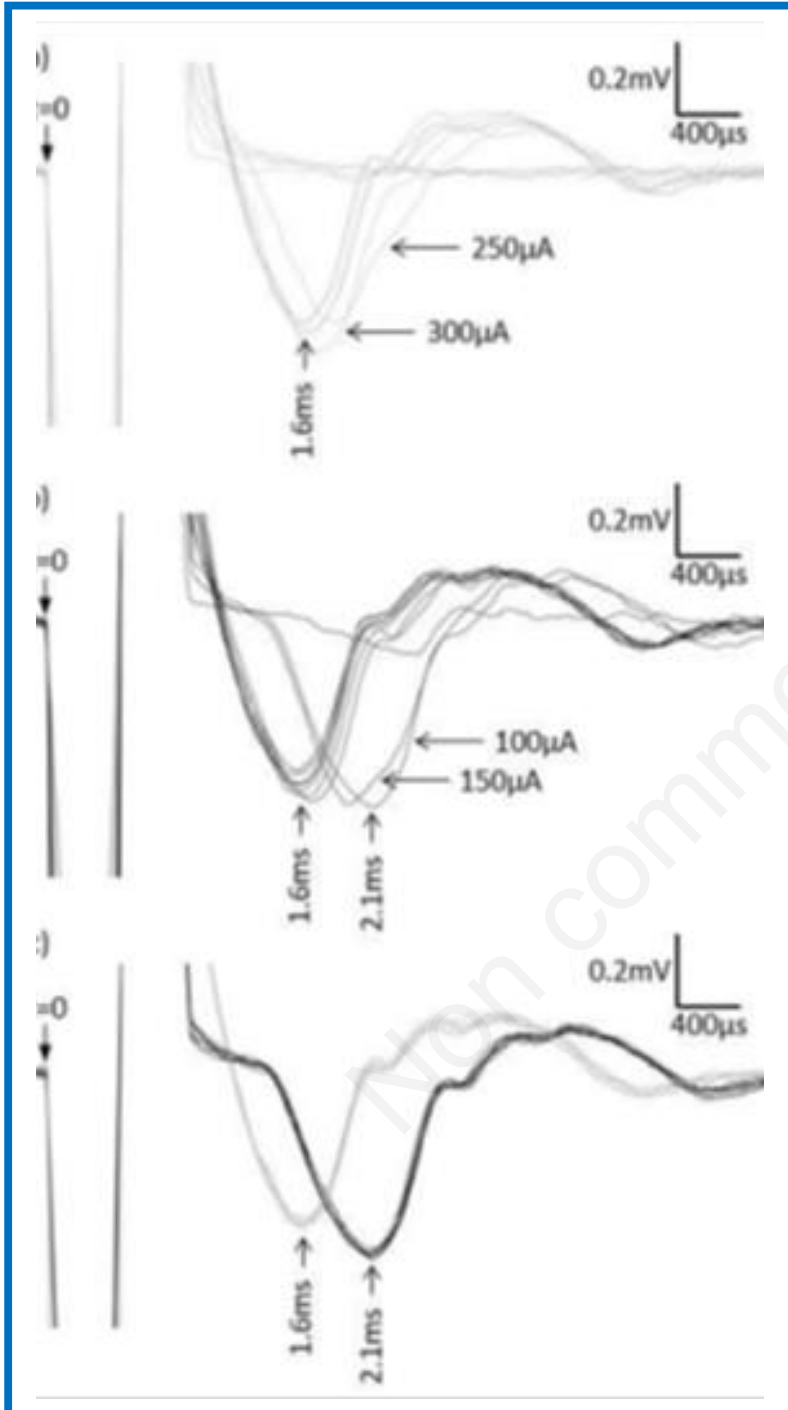

Fig. 2 ENG recordings of the common peroneal nerve during electrical stimulation with $400 \mu$ s phase width pulses. Traces show responses to 50$500 \mu \mathrm{A}$ stimuli (in 50 $\mu \mathrm{A}$ increments) applied via a) monopolar and b) bipolar electrode configurations. c) Eight ENG responses to $400 \mu$ s monopolar stimulation (grey) with 475 $500 \mu A$ compared to eight ENG signals elicited by $400 \mu \mathrm{s}$ bipolar stimuli (black) with 75 $100 \mu A$. electroneurograms (ENG) of the stimulated common peroneal nerve distal to the stimulation electrode(s) as well as the force response of the activated EDL muscle. The main objective was to investigate the delay between the stimulus and the generation of the action in bipolar and monopolar stimulation with a range of stimulus phase widths. Responses to biphasic $400 \mu$ s stimuli applied over a monopolar electrode setup showed a similar delay with the signal peak $1.6 \mathrm{~ms}$ after the stimulation artefact (Figure 2.a). Only pulses that just reached full recruitment showed a slightly longer delay of up to $1.8 \mathrm{~ms}(250 \mu \mathrm{A})$, which might be due to capacitive charging of the tissue. In contrast, Bipolar stimulations with $400 \mu$ s phase width and an amplitude that led to full nerve recruitment resulted in ENG signals with a peakto-artefact delay of $2.1 \mathrm{~ms}$ (Figure 2.b). Further increasing the stimulation amplitude caused a step decrease in the delay to $1.6 \mathrm{~ms}$, similar to the responses to monopolar stimulation. We hypothesize that this jump represents AP generation first at the middle transition and then as the amplitude increases at the first transition of a biphasic stimulus. Figure 2.c compares eight superimposed ENG responses to high amplitude monopolar stimulation (to minimize any time delay due to capacitive charging) with eight superimposed ENG responses to bipolar stimulation at amplitudes below the step in the signal delay. This comparison shows a time difference of approximately $500 \mu \mathrm{s}$, which matches the phase width. This result suggests that there are profound differences in AP generation in monopolar compared to bipolar stimulation that are not yet fully understood or described. As our data on the effect of inter-phase gaps suggested, this difference may have major implications on pulse shaping and practical electrode design.

1. van den Honert C, Mortimer JT. The response of the myelinated nerve fiber to short duration biphasic stimulating currents. Ann Biomed Eng 1979;7:117-25.

2. Gorman PH, Mortimer JT. The Effect of Stimulus Parameters on the Recruitment Characteristics of Direct Nerve Stimulation. IEEE Trans Biomed Eng 1983;30:40714.

3. Reilly JP, Diamant A. Electrostimulation: Theory, Applications, and Computational Model. Artech House Publishers, Boston and London, 2011.

\section{h-bFES of DDM SCI patients and muscle co- activation countermeasures}

\section{Ugo Carraro} Department of Biomedical Sciences, University of
Padova, Italy

\section{E-mail:ugo.carraro@unipd.it}

Spinal cord injury patients spend small amounts of time in daily physical activity and this contributes to further tissue deterioration. ${ }^{1}$ A new wave of papers on "Direct Spinal Cord Stimulation in SCI" promises to revolutionize the management of thoracic-level SCI patients, at least in terms of their mobility. ${ }^{2}$ Unfortunately, there is a group of lumbosacral-level SCI patients, those suffering with a complete irreversible 


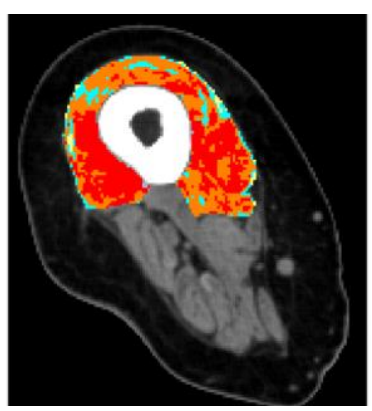

NO h-bFES from 3-y SCl

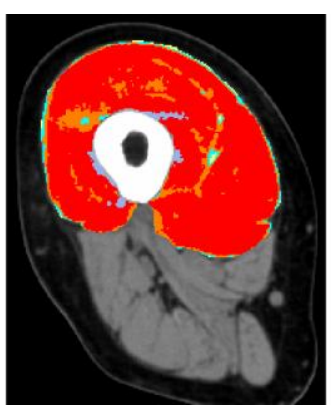

$+2-y$ h-bFES from 3-y SCl

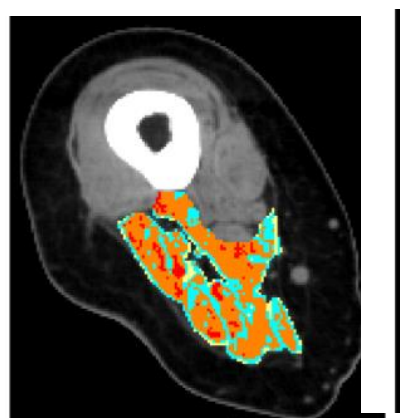

NO h-bFES from 3-y SCl + 2-y h-bFES from 3-y SCl

Fig. 3 Muscle Color Computed Tomography of thigh muscles. It is worth noting that even starting at 3 years post SCI, 2 years of h-bFES is able to induce a substantial recovery of the Hamstring muscles.

conus and cauda equina syndrome, who will never be able take advantage of this break-through management because their leg muscles are permanently disconnected from the Spinal Cord and indeed undergo denervation and degeneration of leg muscles (DDM). ${ }^{3}$ The Vienna Strategy for treatment of DDM is well documented in the previous Reports of RISE - EU Project RISE (Use of electrical stimulation to restore standing in paraplegics with long-term denervated degenerated muscles (QLG5CT-2001-02191). A purpose developed electrical stimulator delivered long impulses of necessary high currents to the thigh muscles by means of large surface electrodes covering the Quadriceps muscle, but indeed this approach also activated the Hamstrings. ${ }^{4}$ Two years of co-activation of the agonist Quadriceps and of the antagonist Hamstrings by home-based functional electrical stimulation (h-bFES) were performed 5 days per week by compliant SCI patients. Twenty out of twenty five patients completed the 2-year h-bFES program, which resulted in a $35 \%$ increase $(p<0.001)$ in cross-sectional area of the Quadriceps muscles. A 75\% increase $(p<0.001)$ in mean diameter of Quadriceps muscle fibers with an improvement of the ultrastructural organization of contractile material and of the $\mathrm{Ca}^{2+}$ handling system occurred together with an impressive $1187 \%$ ( $\mathrm{p}<0.001$ ) increase in force output during electrical stimulation that was sufficient to allow $25 \%$ of patients to perform h-bFES-assisted stand-up and walking-in-place exercises. Interestingly, by muscle color CT scan, ${ }^{5}$ we observed a substantial increase of the Hamstrings, despite these muscles present more atrophy and degeneration at enrolment time (Figure 3). These results were possible applying two large electrodes covering the ventral aspect of the thigh. Thus, continued regularly, h-bFES for DDM helps patients maintain healthier legs and reduces the risks of life-threating SCI complications, even in the worst case of complete and irreversible Conus and Cauda Equina syndrome.

1. Kern H, Carraro U. Home-Based Functional Electrical Stimulation for Long-Term Denervated Human Muscle: History, Basics, Results and Perspectives of the Vienna Rehabilitation Strategy. Eur J Transl Myol 2014;24: 3296. doi: 10.4081/ejtm.2014.3296. eCollection 2014 Mar 31.

2. Angeli CA, Boakye M, Morton RA, et al. Recovery of OverGround Walking after Chronic Motor Complete Spinal
Cord Injury. New Engl J Med 2018;379:1244-1250. doi: 10.1056/NEJMoa1803588. Epub 2018 Sep 24.

3. Kern, H., Carraro U, Adami N, et al. Home-based functional electrical stimulation rescues permanently denervated muscles in paraplegic patients with complete lower motor neuron lesion. Neurorehabil Neural Repair 2010;24: 709-721. doi: 10.1177/1545968 310366129. Epub 2010 May 11.

4. https://www.schuhfried.com/umbraco/Surface/ AuthenticationSurface/Login?return Url $=\% 2$ Fportal

5. Edmunds K, Gíslason M, Sigurðsson S, et al. Advanced quantitative methods in correlating sarcopenic muscle degeneration with lower extremity function biometrics and comorbidities. PLoS One 2018;13: e0193241.

$$
* * * * *
$$

\section{SESSION II: Muscle epigenetics in aging and myopathies}

\section{Lifestyle-related muscle epigenetic memory: mechanisms and biomedical relevance}

\author{
Viviana Moresi \\ Rome Sapienza University, Italy \\ E-mail:viviana.moresi@uniromal.it
}

Current evidence highlights that lifestyle induces alterations to epigenetic modifications leading to changes also across multiple generations, a phenomenon known as intergenerational or transgenerational epigenetic inheritance. Lifestyle factors, including diet, physical activity, tobacco smoking, alcohol consumption, environmental pollutants, psychological stress, perturb the epigenetic landscape influencing the health of several generations in rodents. ${ }^{2,3}$ Most of the studies have been focused on lifestyle factors in relation to DNA methylation, whereas only a few investigations have studied histone modifications and miRNAs. Moreover, chromatin remodeling factors influence the inflammaging, protecting or predisposing an individual to age-related diseases. ${ }^{4}$ Therefore, healthy lifestyles and environmental factors, such as diet interventions or exercise training, are potential biomedical tools to lead to 


\section{Sp PMD Collection of the Abstracts}

Eur J Transl Myol 29 (1):46-78 2019

favorable, heritable epigenetic modifications in order to increase health-protective transcriptional programs.

1. Fleisch AF, Wright RO, Baccarelli AA. Environmental epigenetics: a role in endocrine disease? J Mol Endocrinol 2012;49:R61-7. doi: 10.1530/JME-12-0066.

2. Feil R, Fraga MF. Epigenetics and the environment: emerging patterns and implications. Nat Rev Genet 2012;13:97-109. doi: 10.1038/nrg3142.

3. Abdul QA, Yu BP, Chung HY, et al. Epigenetic modifications of gene expression by lifestyle and environment. Arch Pharm Res 2017;40:1219-37. doi: 10.1007/s12272-017-0973-3. Epub 2017 Oct 17.

4. Szarc vel Szic K, Declerck K, Vidaković M, Vanden Berghe $W$. From inflammaging to healthy aging by dietary lifestyle choices: is epigenetics the key to personalized nutrition? Clin Epigenetics 2015;7:33. doi: 10.1186/s13148-0150068-2. eCollection 2015.

\section{Epigenetics of endurance exercise}

\section{Marco Benati,}

Clinical Biochemistry, University of Verona, Italy

\section{E-mail:marco.benati@univr.it}

Micro RNAs, which are also conventionally known as miRNAs (or miRs), are short non-coding RNA sequences composed of 18-25 nucleotides. Evidence garnered over the past decades demonstrate that miRs play an active role in human muscles, entailing proliferation, differentiation and apoptosis of muscle cells. Their assessment is hence achieving pivotal interest for the diagnosing, prognosticating and following-up skeletal muscle disorders, as well as for better understanding of muscle biology during and after physical exercise. Notably, a highly characteristic array of miRNAs, especially encompassing miRNA-133 and miRNA-206, is specifically expressed within the muscular tissue, thus leading to classify these molecules with the name of myomiRNAs or myomiRs. Recent studies showed that the baseline urine value of both miRNA 133-a and

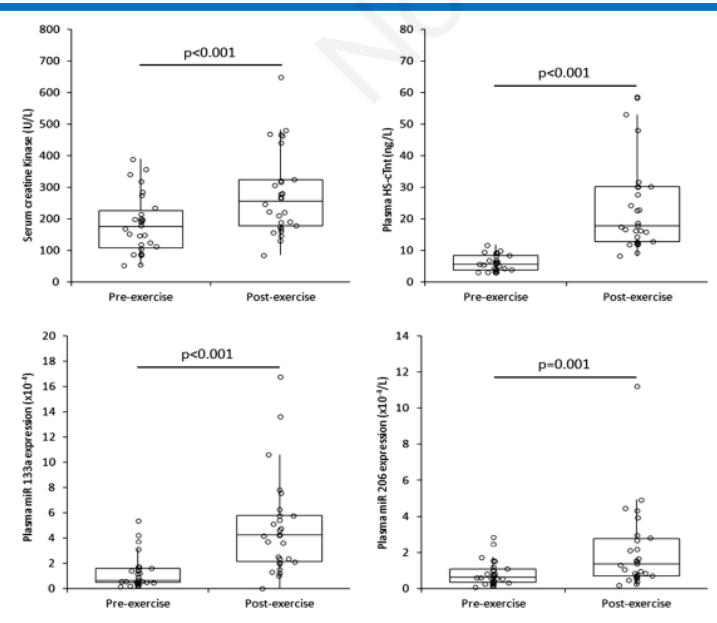

Fig. 4 Variation of serum creatine kinase $(C K)$ and cardiac troponin (cTnT), plasma micro RNA (miR)-133 ${ }^{\circ}$ and $m i R-206$ in 28 athletes (11 women and 17 men).
miRNA 206 is associated with some demographic variables, such as sex (higher in men) and body mass index (BMI), whilst their plasma concentration is independent from the most common physiological variables. Even more importantly, the plasma concentration of both these molecules considerably increases immediately after endurance running, and that their relative increase is highly inter-correlated (Fig. 4). Nevertheless, the relative increase of these two myomiRs does not apparently correlate with any physiological or biochemical parameters (including conventional cardiac and skeletal muscle biomarkers), thus suggesting that the physiological and clinical significance of their variation may reflect a physiological response to high-intensity or prolonged physical exercise, and may also be used for monitoring myogenesis, regeneration or recovery of skeletal muscle following stress, fatigue and muscular exhaustion. Further studies are hence advisable to define as to whether myomiRs may be used along with more conventional laboratory biomarkers for monitoring muscle injury and recovery after physical exercise.

1. Danese E, Benati M, Sanchis-Gomar F, et al. Influence of middle-distance running on muscular micro RNAs. Scand $J$ Clin Lab Invest 2018;78:165-70.

2. Sapp RM, Shill DD, Roth SM, Hagberg JM. Circulating microRNAs in acute and chronic exercise: more than mere biomarkers. J Appl Physiol 2017;122:702-17.

3. Mooren FC, Viereck J, Krüger K, Thum T. Circulating microRNAs as potential biomarkers of aerobic exercise capacity. Am J Physiol Heart Circ Physiol 2014;306:H55763.

4. Lippi $G$ and Plebani M. Personalized medicine: moving from simple theory to daily practice. Clin Chem Lab Med 2015;53: 959-60.

$$
* * * * *
$$

Targeting BET proteins prevents skeletal muscle loss during cancer cachexia

Marco Segatto1, Raffaella Fittipaldi1, Fabrizio Pin 2, Roberta Sartori 4, Kyung Dae Ko 5, Hossein Zare 5, Claudio Fenizia 1, Gianpietro Zanchettin 6, Elisa Sefora Pierobon 6, Shinji Hatakeyama 7, Cosimo Sperti 6, Stefano Merigliano 6, Marco Sandri 3,4, Panagis Filippakopoulos 8, Paola Costelli 2, Vittorio Sartorelli 5, Giuseppina Caretti 1

1 Department of Biosciences, Universita' degli Studi di Milano, Italy; 2 Department of Clinical and Biological Sciences, Unit of General and Clinical Pathology,

University of Turin, Italy; 3 Department of Biomedical Sciences, University of Padova, Padova, Italy; 4Venetian Institute of Molecular Medicine, Padova, Italy; 5 Laboratory of Muscle Stem Cells and Gene Regulation, NIH/NIAMS, Bethesda, MD, US; 6 Department of Surgery, Oncology Gastroenterology, University of Padua, Italy; 7 Musculoskeletal Disease Area, Novartis Institutes for BioMedical Research Basel, Novartis, Basel, Switzerland; 8 Structural Genomics Consortium, Old Road Campus Research Building, Nuffield Department of Medicine, Oxford, UK.

E-mail: giuseppina.caretti@unimi.it 
Skeletal muscle wasting is a hallmark of cancer cachexia. This metabolic syndrome is responsible for about $25 \%$ of cancer deaths. In particular, muscle loss in cachectic patients often leads to increased morbidity and mortality, decreased beneficial effects from chemotherapeutic treatment, and poorer quality of life. Therefore, the development of therapeutic avenues addressed at preventing muscle wasting during cancer cachexia is attracting increasing clinical interest. To date, no effective therapies for cachectic muscle are available. We evaluated the involvement of the epigenetic factor BRD4 in skeletal muscle wasting of cachectic mice. To this aim, C26-tumor bearing mice were chronically treated with the BET inhibitor JQ1 or vehicle. Body weight, skeletal muscle weight and the anabolic/catabolic pathways involved in skeletal muscle homeostasis were analyzed. Our results show that JQ1 treatment blocks musclespecific ubiquitin ligases expression, and protects tumorbearing mice from body weight loss and muscle wasting, without directly affecting tumor growth. Furthermore, JQ1 administration prevents adipose tissue loss and significantly prolongs survival. ChIP-Seq experiments show that the BET protein BRD4 promotes cachexia through activation of the muscle atrophy program. In addition, BET proteins are required to coordinate an IL6dependent AMPK nuclear signaling pathway, converging on the FoxO3 transcription factor. Consistently, BET pharmacological blockade prevents the activation of catabolic genes associated with skeletal muscle atrophy and decreases IL6 systemic levels. Overall, our data uncover that BET proteins may represent a promising therapeutic target to counteract cancer cachexia.

1. Fearon, KC, Glass, DJ, Guttridge, DC. Cancer cachexia: mediators, signaling, and metabolic pathways. Cell Metab 2012;16:153-66.

2. Argilés JM, Busquets S, Stemmler B, López-Soriano FJ. Cancer cachexia: understanding the molecular basis. Nat Rev Cancer 2014;14:754-62.

3. Fearon KC. Cancer cachexia: developing multimodal therapy for a multidimensional problem. Eur J Cancer 2008;44:1124-32.

4. Segatto M., Fittipaldi R, Pin F, et al Epigenetic Targeting of Bromodomain Protein BRD4 Counteracts Cancer Cachexia and Prolongs Survival. Nature Comm 2017;8(1):1707.

\section{Session III: Experimental approaches in animal models}

\section{The ERG1a $\mathrm{K}^{+}$channel increases calpain activity in C2C12 myotubes and mouse skeletal muscle}

Amber L. Pond 1, Clayton Whitmore 1, Evan E. Pratt 2, Luke Anderson 1, Emily Rantz 2, Amy Salyer 2, Ugo Carraro 3, Wen-Horng Wang 4, Rod Weilbaecher 5, Judy K. Davie 5, Gregory H. Hockerman 2

1 Anatomy Dept., Southern Illinois Univ. School of Medicine, Carbondale, IL; 2 Medicinal Chemistry and Molecular Pharmacology Dept., Purdue Univ. School of Pharmacy, West Lafayette, IN; 3 Biomedical Dept., University of Padova, Italy; 4 Gene Editing Core Facility, Purdue University, West Lafayette, IN; 5 Biochemistry Dept., Southern Illinois Univ. School of Medicine, Carbondale, IL, USA

E-mail: apond@siumed.edu

Increased intracellular calcium concentration $\left(\left[\mathrm{Ca}^{2+}\right] \mathrm{i}\right.$; $\mathrm{uM}$ range) is necessary for excitation-contraction coupling in skeletal muscle; however, smaller increases in $\left[\mathrm{Ca}^{2+}\right] \mathrm{i}$ (nM range) can act as a second messenger to modulate other cellular processes in non-contracting muscle (Figure 5). In contrast, improper changes in $\left[\mathrm{Ca}^{2+}\right] \mathrm{i}$ can have detrimental effects on muscle and have been associated with pathologies, for example muscular dystrophy, amyotrophic lateral sclerosis, malignant hyperthermia, cancer cachexia, and atrophy. ${ }^{1-4}$ Indeed, $\left[\mathrm{Ca}^{2+}\right] \mathrm{i}$ must be rigidly regulated in terms of time, space and amplitude for cellular processes to occur in a properly coordinated fashion.1 In earlier reports, we have shown that the ether-a-gogo related gene (ERG) 1a $\mathrm{K}^{+}$channel is detected in atrophying skeletal muscle and that mouse erg1a (Merg1a) expression in mouse muscle upregulates ubiquitin proteasome proteolysis. ${ }^{5,6}$ Because ERG1a is detected in the t-tubules of cardiac muscle, ${ }^{7}$ we

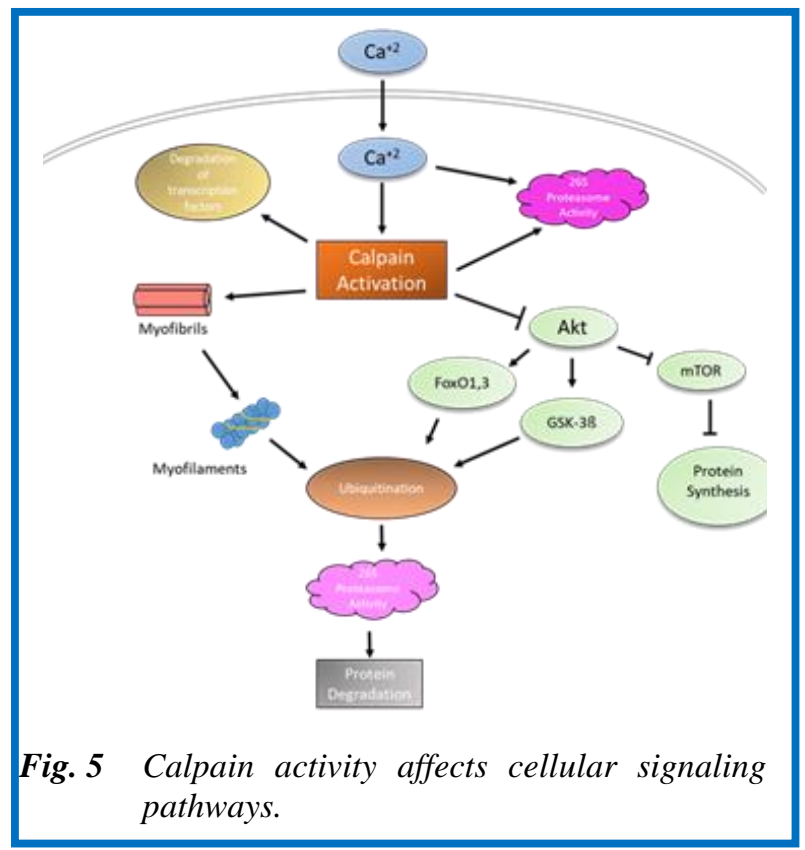




\section{Sp PMD Collection of the Abstracts}

Eur J Transl Myol 29 (1):46-78 2019

hypothesized that it would affect intracellular calcium concentration and thus activity of calpains (calcium activated cysteine proteases known to contribute to protein loss in atrophy). We tested this hypothesis using the ratiometric $\mathrm{Ca}^{2+}$ indicator fura-2AM, and here show that viral transduction of human ERG1a (HERG1a) into C2C12 myotubes for 48 hours produces a $34 \%$ increase in basal intracellular calcium levels $\left(\left[\mathrm{Ca}^{2+}\right] \mathrm{i}\right)$ relative to myotubes transduced with the appropriate control virus ( $\mathrm{p}<0.0001 ; \mathrm{n}=87$ HERG1A; $\mathrm{n}=90$ control). Further, we transduced myotubes with HERG1a and, using a CalpainGlo assay kit (ProMega; Madison, WI), determined that the combined calpain 1 and 2 activity increased by $25.7 \%$ relative to controls. The change in calpain activity is likely attributable to increased calcium levels; however, other explanations include increased calpain protein resulting from enhanced transcription and/or translation or decreased calpain protein degradation. Further, decreased abundance of the native inhibitor calpastatin could explain enhanced calpain activity. Thus, we performed quantitative PCR on myotubes transduced with control and HERG-encoded virus for 48 hours and detected no significant changes in expression of calpains 1,2 and 3 nor of calpastatin. Immunnoblot detected no change in calpain 1 protein abundance. However, immunoblot revealed a $40.7 \%$ decrease in abundance of calpain 2 protein and a $23.5 \%$ decrease in abundance of calpain 3 . Obviously, these data do not explain the increase in calpain activity and, indeed, the increased degradation of the calpain proteins may be attributable to the increased calpain activity produced by the increased calcium concentration. Further, calpastatin protein abundance also decreased by $37.7 \%$. This lowered level of calpain inhibitor could indeed contribute to the increased calpain activity. Interestingly, we electro-transferred Merg1a encoded plasmid into mouse Gastrocnemius muscles and at day 4 post-electro-transfer detected a 7-fold increase in calpain activity over control in homogenated samples. Because the cellular structure had been disrupted in this experiment, the increase in calcium concentration cannot be the sole contributor to the increased calpain activity. Indeed, the decreased calpastatin protein levels may be important.

1 Costelli P, Reffo P, Penna F, Autelli R, Bonelli G, Baccino FM. Ca2+-dependent proteolysis in muscle wasting. Int $J$ Biochem Cell Biol 37:2134-2146, 2005.

2 Goswami A, Jesse CM, Chandrasekar A, Bushuven E, Vollrath JT, Dreser A, Katona I, Beyer C, Johann S, Feller AC, Grond M, Wagner S, Nikolin S, Troost D, Weis J. Accumulation of STIMI is associated with the degenerative muscle fibre phenotype in ALS and other neurogenic atrophies. Neuropath Appl Neurobiol 41:304-318, 2015.

3. Missiaen L, Robberecht W, van Den Bosch L, callewaert $G$, Parys JB, Wuytack F, Raeymaekers L, Nilius B, Eggermont $J$, De Smedt H. Abnormal intracellular calcium homeostasis and disease. Cell Calcium 28:1-21, 2000.

4 Mukherjee S, Brooks WH. Stromal interaction molecules as important therapeutic targets in diseases with dysregulated calcium flux. Biochim biophys Acta 1843:2307-2314, 2014.
5. Pond $A L$, Nedele $C$, Wang $W$ - $H$, Wang $X$, Walther $C$, Jaeger $C$, et al. The MERGla channel modulates skeletal muscle Murfl, but not MAFbx, expression. Muscle \& Nerve. 2013;49(3):378-388.

6. Wang X, Hockerman GH, Green 3rd HW, Babbs CF, Mohammad SI, Gerrard D, Latour MA, London B, Hannon KM, Pond AL. Mergla K+ channel induces skeletal muscle atrophy by activating the ubiquitin proteasome pathway. FASEB J 20(9):1531-3, 2006.

7. Rasmussen HB, Moller M, Knaus H-G, Jensen BS, Olesen $S-P$, Jorgensen NK. Subcellular localization of the delayed rectifier K+ channels KCNQ1 and ERG1 in the rat heart. Am J Physiol Heart Circ Physiol. 2004;286:H1300-H1309.

$$
* * * * *
$$

\section{Vgll3 interacts with Yap/Taz-Tead1-4-dependent gene expression in skeletal muscle}

Nicolas Figeac, Mohamed Abdalla, Congshan Sun, Martin Schönfelder, David Gomez, Amaya GarciaMunoz, Edoardo Missiaglia, Elaina Collie-Duguid, Vanessa De Mello, Ajaybabu V. Pobbati, Oihane Jaka, Stephen DR Harridge, Wanjin Hong, Janet Shipley, Neil Vargesson, Pete Zammit, Henning Wackerhage

Technical University of Munich, Faculty of Sport and Health Sciences, Munich, Germany

\section{E-mail: henning.wackerhage@tum.de}

Vgll3 is a transcriptional co-factor that is expressed at low levels in differentiated skeletal muscle but whose expression increases in response to challenges and in human muscle-related disease such as Duchenne muscular dystrophy and alveolar rhabdomyosarcoma. To study whether and how Vgll3 interacts with Tead family transcription factors, we have expressed and coimmunoprecipitated Vgll3-flag in C2C12 myoblasts and myotubes and identified binding partners through liquid chromatography followed by mass spectrometry. Additionally, we have expressed Vgll3 in myoblasts and measured the effect on gene expression. We found that Vgll3 binds the same Tead1,3,4 transcription factors as Yap and Taz and that Vgll3 mainly represses genes. Vgll3 regulates the expression of many Yap and Taz regulated genes either as a YAP antagonist or it corepresses the same genes that are repressed by YAP. Together this identifies Vgll3 as a transcriptional cofactor that is induced in response to challenges and diseases and that interferes with Yap/Taz-Tead1-4mediated gene expression in the muscle lineage.

\section{Genes whose gain or loss-of-function increases endurance performance in mice: a systematic literature review}

Fakhreddin Yaghood Nezdad, Sander Verbrugge, Martin Schönfelder, Lore Becker, Martin Hrabě de Angelis, Henning Wackerhage

Technical University of Munich, Faculty of Sport and Health Sciences, Munich, Germany

E-mail: henning.wackerhage@tum.de 
Endurance is not only a key factor in many sports but endurance-related variables are also associated with good health and low mortality. Twin and family studies suggest that several endurance-associated traits are $\square 50 \%$ inherited. However, we still poorly understand what DNA sequence variants contribute to endurance heritability. To address this issue, we conducted a systematic review to identify genes whose experimental loss or gain-of-function increases endurance capacity in mice. We found 31 genes including two isoforms of Ppargcla whose manipulation increases running or swimming endurance performance by up to $1800 \%$. Genes whose gain-of-function increases endurance are Adcy5, Adcy8, Hk2, Il15, Mef2c, Nr4a3, Pck1 (Pepck), Ppard, Ppargc1a (both the $\mathrm{a}$ and $\mathrm{b}$ isoforms of the protein Pgc-1 $\alpha$ ), Ppargc1b, Ppp3ca (calcineurin), Scd1, Slc5a7, Tfe3, Tfeb, Trib3 \& Trpv1. Genes whose loss-offunction increases endurance in mice are Actn3, Adrb2, Bdkrb2, Cd47, Crym, Hif1a, Myoz1, Pappa, Pknox1, Pten, Sirt4, Thbs1, Thra \& Tnfsf12. Of these genes, human DNA sequence variants of ACTN3, ADCY5, ADRB2, BDKRB2, HIF1A, PPARD, PPARGC1A, PPARGC1B and PPP3CA are also associated with endurance capacity and/or $\mathrm{VO} 2 \mathrm{max}$ trainability suggesting evolutionary conservation between mice and humans. Bioinformatical analyses show that there are numerous amino acid or copy number-changing DNA variants of endurance genes in humans, suggesting that genetic variation of endurance genes may contribute to the variation of human endurance capacity, too. Moreover, several of these genes/proteins change their expression or phosphorylation in skeletal muscle or the heart after endurance exercise, suggesting a role in the adaptation to endurance exercise.

$$
* * * * *
$$

\section{High intensity exercise induces muscle remodeling through chromatin modifications and increased MRTF-SRF signaling}

Francesca Solagna, Leonardo Nogara, Kenneth A. Dyar, Henriette Uhlenhaut, Kristian Vissing, Marcus Kruger, Bert Blaauw

\section{Department of Biomedical Sciences, University of Padova, Italy}

\section{E-mail:bert.blaauw@unipd.it}

Muscle plasticity after exercise is achieved by different molecular mechanisms that regulate gene transcription by impinging on chromatin structure and transcription factors. We performed an unbiased quantitative phosphoproteomics approach in vivo in order to determine the early signaling changes occurring after high-intensity exercise. We identified two histone modifications, which are strongly increased after exercise and potentially linked to increased gene transcription. CHIP analysis confirmed that H3S10 phosphorylation happens on SRF-dependent genes like Fos, Jun and Egr1. Furthermore, we identify a completely new phosphorylation of MRTF-B, a transcriptional coactivator of SRF-dependent gene transcription, which is critical for its nuclear localization. Based on our results, we are proposing a model in which histone modifications and nuclear translocation of MRTF-B act in concert for the activation of SRF-dependent gene transcription, a well-known mediator of exercise-induced muscle remodeling.

$$
* * * * *
$$

\section{Polyglutamine-expanded androgen receptor in SBMA leads to altered excitation-contraction coupling and muscle force production before motor nerve dysfunction}

Marco Pirazzini 1,2, Marchioretti C 1,2, Chivet M 3, Piol D 1,2, Scaramuzzino C 4, Polanco JM 3, Nath S 5, Zuccaro E 1,2, Nogara L 1,2, Armani A 2, Parodi S 4, D'Antonio M 5, Megighian A 1, Sambataro F 6, Pegoraro E 7, Sorarù $G 7$, Rinaldi $C 8$, Lieberman AP 5, Blaauw B 1,2,9, Sandri M 1,2,9, Basso M 10, PennutoM 1,2,3,9,11

1. Department of Biomedical Sciences, University of Padova, Padova, Italy; 2 Venetian Institute of Molecular Medicine, Padova, Italy; 3 Dulbecco Telethon Institute, Centre for Integrative Biology, University of Trento, Trento, Italy; 4 Department of Neuroscience and Brain Technologies, Istituto Italiano di Tecnologia, 16163 Genova, Italy; 5 Division of Genetics and Cell Biology, San Raffaele Scientific

Institue, Milan, Italy; 6 Department of Experimental \& Clinical Medical Sciences (DISM), University of Udine, Udine, Italy; 7 Department of Neurosciences, University of Padova, 35100 Padova, Italy; 8 Department of Physiology, Anatomy and Genetics, University of Oxford, OXI 3QX Oxford, UK; 9 Myology Center, University of Padova, 35100 Padova, Italy; 10 Centre for Integrative Biology, University of Trento, Trento, Italy; 11. Padova Neuroscience Center, Padova, Italy

E-mail: marco.pirazzini@unipd.it

Spinal and bulbar muscular atrophy (SBMA), also known as Kennedy disease, is an X-linked neuromuscular disease caused by polyglutamine (polyQ) expansions in the gene encoding androgen receptor (AR), which is a transcription factor regulating gene expression in response to androgen binding. PolyQ expansion confers both loss of function (LOF) and toxic gain of function (GOF) to AR. SBMA is characterized by primary pathogenetic processes occurring in lower motor neurons as well as in the innervated skeletal muscles. Although clinical and experimental evidence highlight a primary role for skeletal muscle in the onset, progression and outcome of disease, the pathophysiological and molecular processes underlying SBMA muscle atrophy are poorly understood. Here we provide the first evidence that polyQ expansions in the AR disrupts the excitation-contraction coupling (ECC) machinery and alters calcium dynamics in skeletal muscle, and that these pathological processes occur in the absence of functional denervation. We show that the expression of ECC genes is altered through a mechanism that involves a loss of AR signaling. Finally, we provide evidence that ECC gene expression alterations can be restored by pharmacologic treatment of SBMA mice that suppresses polyQ- 


\section{Sp PMD Collection of the Abstracts}

Eur J Transl Myol 29 (1):46-78 2019

expanded AR toxicity. These observations imply a model whereby AR regulates muscle function by controlling muscle contraction, and deregulation of AR signaling leads to alteration of the ECC machinery, calcium dyshomeostasis and muscle weakness and atrophy.

$$
* * * * *
$$

\section{Mitochondrial adaptation in parvalbumin knockout muscle fibres}

Gaia Butera, Marta Canato, D Vecellio Reane, Rosario Rizzuto, A Raffaello, Carlo Reggiani

Department of Biomedical Sciences, University of Padova, Padua, Italy

\section{E-mail: gaia.butera@studenti.unipd.it}

In skeletal muscle, mitochondrial $\mathrm{Ca}^{2+}$ uptake plays important roles in organ homeostasis. In order to understand the importance of mitochondria in cytosolic $\mathrm{Ca}^{2+}$ buffering, we explored the effects of removing parvalbumin (PV), the most important cytosolic $\mathrm{Ca}^{2+}$ buffer. By using a PV knockout (KO) mouse model, we are investigating whether the absence of PV induces a compensatory mechanism on the expression and function of the mitochondrial $\mathrm{Ca}^{2+}$ uptake machinery (MCU complex). The data collected so far confirm that the absence of PV induces an increase of mitochondrial $\mathrm{Ca}^{+}$ uptake accompanied by a profound adaptation of the expression of the MCU complex components. Furthermore, cytosolic $\mathrm{Ca}^{2+}$ transients in PV KO muscle fibres show that the time to reach the peak upon stimulation and the time to half relaxation are prolonged in PV KO muscle fibres in comparison with WT animals, given to a major fatigue resistance and ability to sustain muscle activity for prolonged period of PV KO mice. To verify whether the increase of mitochondrial $\mathrm{Ca}^{2+}$ uptake in PV KO fibers is due to mitochondrial adaptation and whether MCU is responsible of buffering cytosolic calcium increases, we decided to silencing MCU and measure $\left[\mathrm{Ca}^{2+}\right]$ cyt evoked by caffeine, that induces the release of $\mathrm{Ca}^{2+}$ from the SR. FDB muscles of WT and PV KO animals were transfected in vivo with plasmids encoding either control shRNA (shLuc) or shRNA designed to specifically silence MCU (shMCU). One week after, the fibers were dissected and loaded with Fura-2/AM. $\left[\mathrm{Ca}^{2+}\right]$ cyt was almost unaffected by the absence of MCU in WT animal, while in PV KO fibers, $\left[\mathrm{Ca}^{2+}\right]$ cyt was significantly higher upon stimulation. This data data play in favour of the view that mitochondria in PV KO mice adapt to buffer $\left[\mathrm{Ca}^{2+}\right]$ cyt. Intriguingly, since $\mathrm{PV}$ is one of the most downregulated atrogenes, the genes modulated during several types of atrophy, and that mitochondrial $\mathrm{Ca}^{2+}$ controls skeletal muscle tropism, we decided to study the role of PV on muscle trophism through denervation experiments on $\mathrm{PV} \mathrm{KO}$ mice. Denervation atrophy was triggered by sciatic nerve section and fibres size was evaluated. When PV is absent, loss of muscle mass was reduced compared to innervated control fibres demonstrating that PV can partially protect muscles from denervation-induced atrophy. Silencing experiments gave coherent results. Tibialis anterior muscles of WT were transfected in vivo with plasmids encoding either shLuc or shPV. Fibres positive for shPV are significantly larger than fibres expressing the shLuc control plasmid. In conclusion, our results indicate that PV plays an important role in spatiotemporal control of cytosolic $\mathrm{Ca}^{2+}$ responses on mitochondrial $\mathrm{Ca}^{2+}$ uptake and have a profound impact on skeletal muscle trophism.

$* * * * *$

\section{Novel zebrafish models of Sarcoglycanopathy}

Michela Soardi 1, Marcello Carotti 1, Cristiano Bertolucci 2, Giovanni Risato 1, Martina Scano 1, Francesco Argenton 3, Roberta Sacchetto 4, Dorianna Sandonà 1

1 Department of Biomedical Sciences, University of Padova, Padova, Italy; 2 Department of Life Sciences and Biotechnologies, University of Ferrara, Italy; 3 Department of Biology, University of Padova, Padova, Italy; 4 Department of Comparative Biomedicine and

Food Science, University of Padova, Padova, Italy

\section{E-mail: dorianna.sandona@unipd.it}

Sarcoglycanopathy is a rare, progressive, autosomal recessive myopathy belonging to the Limb Girdle Muscular Dystrophy Family because of the major involvement of the proximal musculature of pelvic and scapular girdle. This still incurable disease is caused by mutation in SGCA, SGCB, SGCG, SGCD, genes coding for alpha-, beta-, gamma- and delta-sarcoglycan (SG), respectively. SGs are transmembrane glycoproteins forming a sub-complex that is part of the dystrophin associated glycoprotein complex (DAPC), fundamental to protect the plasma membrane of striated muscle from contraction stresses. ${ }^{1,2}$ Most of the SG defects are missense mutations resulting in folding-defective proteins recognised by the quality control systems (QCS) of the cell and degraded by the proteasome, as substrates of the endoplasmic reticulum associated degradation (ERAD) pathway. This event causes the secondary deficiency of the wild type partners, the alteration of the DAPC and the consequent progressive degeneration of striated muscle. ${ }^{3}$ Recently, it was described a therapeutic approach, based on small-molecules able to recover the SG-complex. Specifically, by blocking different steps of the ERAD pathway, additional rounds of folding are forced and the misfolded mutant can acquire a native like conformation and assemble with the other partners at the sarcolemma. This means that the loss of function is due to a premature degradation and not to a functional impairment of the mutant. ${ }^{4}$ Moreover, it is also possible to help the folding of the protein by using small molecules known as CFTR correctors, acting as chaperones or more probably as proteostasis regulators. The proof of concept of these pharmacological approaches have been established in vitro using cell models and, notably, myogenic cells from a LGMD2D patient. ${ }^{5}$ To move to an effective therapy, it is necessary to evaluate efficacy of the small molecules in vivo. To this purpose, we are now generating novel models of sarcoglycanopathy in D. rerio (zebrafish). The choice felt on this small vertebrate, because, in the absence of suitable rodent models, zebrafish is an excellent model 


\section{Sp PMD Collection of the Abstracts}

Eur J Transl Myol 29 (1):46-78 2019

for studying muscular disorders, to perform drug screening, and for genome-editing experiments. Here we show first, the proof of concept of modelling sarcoglycanopathy in zebrafish taking advantage by the morpholino (MO) knock down (KD) of delta-SG. Then, confident by the promising results obtained, we describe the generation of stable Knock out (KO) of beta- and delta-SG by CRISPR/Cas9 genome editing. The KO mutants are used to better characterize the pathology and as background for the injection of either the WT or mutated SG mRNAs. The injection of WT sequence is used to rescue the null-phenotype, while the mutated sequences should result in the development of pathologic features. Thanks to this strategy, we have the possibility to study a significant number of different mutations thus evaluating the wideness of applicability of CFTR correctors in sarcoglycanopathy. We are also generating beta-SGT145R/T145R and delta-SGE264K/E264K zebrafish KI that, once characterized, will allow for direct testing of small molecules. The novel $\mathrm{KO}$ and $\mathrm{KI}$ zebrafish models will represent a fundamental tool to test our pharmacological approach and will be a valuable adjunction to the already available vertebrate models of sarcoglycanopathy for both basic and translational research.

1. Kirschner J, Lochmuller H. Sarcoglycanopathies. Handb Clin Neurol 2011;101:41-6.

2. Carotti M, Fecchio C, Sandona D. Emerging therapeutic strategies for sarcoglycanopathy. Expert Opin Orphan D 2017;5:381-96.

3. Gastaldello $S$, D'Angelo $S$, Franzoso $S$, et al. Inhibition of proteasome activity promotes the correct localization of disease-causing alpha-sarcoglycan mutants in HEK-293 cells constitutively expressing beta-, gamma-, and deltasarcoglycan. Am J Pathol 2008;173:170-81.

4. Bianchini E, Fanin M, Mamchaoui K, Betto R, Sandona D. Unveiling the degradative route of the V247M alphasarcoglycan mutant responsible for LGMD-2D. Hum Mol Genet 2014;23:3746-58.

5. Carotti M, Marsolier J, Soardi M, et al. Repairing foldingdefective alpha-sarcoglycan mutants by CFTR correctors, a potential therapy for limb-girdle muscular dystrophy $2 D$. Hum Mol Genet 2018;27:969-84.

$$
* * * * *
$$

\section{Neutral Lipid Storage Disease with Myopathy: functional study to clarify pathogenic effect of ATGL mutations}

Sara Missaglia 1,2, Corrado Angelini 3, Elena Maria Pennisi 4, Marina Mora 5, Daniela Tavian 1,2

1 Laboratory of Cellular Biochemistry and Molecular Biology, CRIBENS, Catholic University of the Sacred Heart, Milan, Italy; 2 Psychology Department, Catholic University of the Sacred Heart, Milan, Italy; 3 IRCCS

Fondazione Ospedale S. Camillo, Venice, Italy; 4 UOC

Neurologia, San Filippo Neri Hospital, Italy: 5

Neuromuscular Diseases and Neuroimmunology,

Fondazione IRCCS Istituto Neurologico "Carlo Besta”, Milan, Italy

E-mail: daniela.tavian@unicatt.it
Neutral Lipid Storage Disease with Myopathy (NLSDM) is a rare autosomal recessive disorder characterized by an abnormal accumulation of triacylglycerol into cytoplasmic lipid droplets (LDs). The most important symptom of NLSDM is the progressive skeletal muscle myopathy. ${ }^{1}$ The presence of lipid inclusions in muscle biopsies and elevated serum transaminases and creatine kinase (CPK) suggests a muscle damage. In particular, a consistently reported characteristic of the NLSDM is the progressive muscular atrophy. Other clinical symptoms may include cardiomyopathy, diabetes, chronic pancreatitis and short stature. The severity of these clinical features appears to be highly variable. ${ }^{1}$ The onset of NLSDM has been associated with PNPLA2 mutations. This gene codes for adipose triglyceride lipase (ATGL), an enzyme that hydrolyses fatty acids from triacylglycerol. This lipase comprises two functional regions: a patatin domain, that contains the catalytic site (S47 and D166), and a LDs-binding site. Until now, fiftyfive patients harboring thirty-nine different mutations have been reported. Twenty-two of these variations result in premature stop codons and determine the formation of truncated proteins, four are nonsense mutations leading to complete absence of ATGL production and thirteen are missense mutations. ${ }^{1}$ To determine the pathogenic effect of PNPLA2 missense mutations identified in NLSDM patients, we performed functional studies. ${ }^{2-4}$ Seven missense variations were investigated: two were located in the patatin domain (L56R and N172K), one affected the catalytic site (D166G), three were placed between the patatin and hydrophobic regions (I193F, P195L and $\mathrm{R} 221 \mathrm{P}$ ), and one was located in the C-terminal site (P481L). HeLa cells were transiently transfected with different recombinant plasmid, with GFP at C-terminus and containing either human ATGL wild type or ATGL mutant cDNA. Before the transfection, the cells were incubated with $400 \mu \mathrm{M}$ oleic acid (OA) bound to bovine serum albumin (BSA). All mutant proteins correctly localized to LDs surface, but the analysis of LDs number and dimensions showed that, compared the control vector, the mutant proteins ATGL(L56R), ATGL(N172K), and ATGL(I193F) presented a partial lipase activity, while the activity of ATGL(P195L) and ATGL(R221P) proteins was severely impaired. ATGL(P481L) showed a slight decrease of lipase activity. Finally, ATGL(D166G) protein was totally inactive. The complete loss of ATGL activity causes a severe defect of LD metabolism and an abnormal accumulation of neutral lipid in skeletal muscle and heart, determining the onset of myopathy and cardiomyopathy [5]. By contrast, mutations that maintain a minimal ATGL activity may be associated with a slowly progressive myopathy and preserve heart function. ${ }^{3-5}$ Data obtained from these studies demonstrate that the evaluation of functional effects of mutations located in the different domains of ATGL may be important to provide a more accurate disease prognosis.

1. Pennisi EM, Arca M, Bertini E, et al. Neutral Lipid Storage Diseases: clinical/ genetic features and natural history in a large cohort of Italian patients. Orphanet $J$ Rare Dis 2017;12:90. 
2. Tavian D, Missaglia S, Redaelli C, et al. Contribution of novel ATGL missense mutations to the clinical phenotype of NLSD-M: a strikingly low amount of lipase activity may preserve cardiac function. Hum Mol Genet 2012;21:5318-28.

3. Pennisi EM, Missaglia S, Di Mauro S, et al. A myopathy with unusual features caused by PNPLA2 gene mutations. Muscle Nerve 2015;51 609-13.

4. Missaglia S, Tasca E, Angelini C, et al. Novel missense mutations in PNPLA2 causing late onset and clinical heterogeneity of neutral lipid storage disease with myopathy in three siblings. Mol Genet Metab 2015;115:110-7.

5. Coassin S, Schweiger M, Kloss-Brandstätter A, et al. Investigation and functional characterization of rare genetic variants in the adipose triglyceride lipase in a large healthy working population. PLoS Genet 2010;6:e1001239.

$* * * * *$

\section{March 29, 2019 - Botanical Garden, University of Padova, Italy}

\section{Three Physiology Lectures}

\section{Na Channel Activation and Inactivation: Role of Domain 4}

Clay Armstrong

Penn University, Philadelphia, Pa, USA

\section{E-mail: carmstro@pennmedicine.upenn.edu}

The Na channel has transmembrane domains DI through D4. Following Hodgkin and Huxley, it is often thought that the DI thru D3 are involved in activation of the channel, and D4 is devoted exclusively to inactivation. Based on the literature, we believe instead that D4 is involved in both activation and inactivation. Sheets et al. (2015) showed that in Nav1.4 the D4S6 mutation $\mathrm{N} 1584 / \mathrm{C}$ eliminates $\mathrm{gNa}$ without disturbing charge movement; i.e., all of the voltage sensing S4's move normally (normal gating current), but the channel does not open; showing clearly that D4 participates in activation. We postulate that the open (conducting) state is stabilized by H-bonding of N1584 (in S6 of D4 near the gate region) to $\mathrm{S} 1471$ at the S45-S5 junction (c.f. N408 and S320 of the K channel structure 2R9R). Many $\mathrm{Na}$ channels have similarly located $\mathrm{N}$ (in S6) and S (in $\mathrm{S} 45$ ) in all four domains. Turning to the S4 segment of D4, we believe activation requires S4R4 in QTCin (inner charge transfer center), and that inactivation becomes possible when it moves thru the membrane field to QTCout; in this condition IFM (in S6) can enter and inactivate the channel (Kuhn\&Greef). Recovery requires that S4R4 move from QTCout to QTCin, forcing IFM out of the channel. A second, more stable inactivated state requires that S4K6 enter QTCin, to which K6 binds more stably than R5. Leak is prevented during recovery by quick return to rest of the S4's of D1 \&D2, which are not immobilized by inactivation.

\section{Bench to bedside research on Critical Illness Myopathy and Ventilator Induced Diaphragm Muscle Dysfunction}

\section{Lars Larsson}

\author{
Department of Physiology \& Pharmacology, \\ Department of Clinical Neuroscience, Clinical \\ Neurophysiology, Karolinska Institutet, Stockholm, \\ Sweden
}

\section{E-mail: lars.larsson@ki.se}

Acquired paralyses of limb and trunk muscles (critical illness myopathy, CIM) are common in intensive care unit (ICU) patients exposed to prolonged mechanical ventilation and immobilization. CIM is attendant to essential and appropriate lifesaving interventions in modern ICUs; but, the inadvertent myopathic consequences of extended mechanical ventilation increase morbidity and/or mortality; have severe impact on the patient's quality of life, and burden the health care system with staggering costs. Despite this, there is currently no treatment available for CIM apart for symptomatic and supportive interventions. Neuromuscular blockade, massive doses of steroids and sepsis have been forwarded as critical factors triggering CIM/VIDD, but clinical and experimental work from our group show that none of these factors are obligatory in the pathophysiology of CIM/VIDD. Long-term experiments are vital to our understanding of mechanisms and the evaluation of interventions since it takes longer than 5 days exposure to the ICU condition (mechanical ventilation and immobilization) to develop the hallmark of CIM in both humans and rats, i.e., the preferential loss of the molecular motor protein myosin in limb and trunk muscles. Long-term experiments using a novel experimental rodent ICU model used in our lab have shown that rats develop the same geno-/pheno-type observed in ICU patients with CIM in response to longterm exposure to the ICU condition. This model is accordingly well suited for mechanistic studies as well as the evaluation of specific intervention strategies targeting underlying mechanisms prior to the translation to the clinic. The long-term scientific goals of our research are to i) understand the mechanisms underlying the severely impaired limb muscle function associated with CIM, ii) develop sensitive, quick and accurate methods for clinical diagnosis and monitoring, and iii) evaluate different interventions strategies in the ExICU model and translate promising interventions to clinical research. In this presentation, specific intervention strategies targeting the mechanisms underlying CIM and VIDD and their potential translation to the clinic will be presented.

$$
* * * * *
$$

\section{Measuring mitochondrial calcium in muscle fibers at rest and during contraction}

Carlo Reggiani, Marta Canato, Lorenzo Marcucci Department of Biomedical Sciences, University of
Padova, Padua, Italy

E-mail: carlo.reggiani@unipd.it 
Mitochondrial free calcium concentration $\left(\left[\mathrm{Ca}^{2+}\right]\right.$ mito) regulates mitochondrial energy production, provides transient $\mathrm{Ca}^{2+}$ buffering under stress, and can be involved in apoptosis and cell death. In skeletal muscle fibers, mitochondria are located near the calcium release units (CRU) of the sarcoplasmic reticulum (SR), and evidence exists for crosstalk between the two organelles. Direct quantitative measurements of $\left[\mathrm{Ca}^{2+}\right]$ mito in skeletal muscle fibers are limited, and the contribution of mitochondria to intracellular calcium homeostasis is still debated. Using cameleon $4 \mathrm{mtD} 3 \mathrm{cpv}$ as a calcium indicator targeted to mitochondria matrix and calibrated in situ, we directly measured $\left[\mathrm{Ca}^{2+}\right]$ mito in intact adult muscle fibers dissociated from murine FDB with a time resolution of $7 \mathrm{~ms}$ (Scorzeto et al. 2013, Marcucci et al. 2018). At rest, the levels of free calcium concentration are similar in mitochondrial matrix and in the cytosolic space. When calcium release from SR is triggered by electrical stimulation or by chemical stimulation with caffeine, $\left[\mathrm{Ca}^{2+}\right]$ mito increases and reaches levels higher than the peak of average cytosolic calcium, as can be measured with Fura 2. This can be explained by the specific localization of the mitochondria close the calcium release units and the electrochemical gradient driving calcium entrance via MCU. A pronounced asymmetry between the rising phase and the declining phase is a peculiar feature of the intra-mitochondrial calcium transient during an event of calcium release from SR. As a direct consequence of the slow decay phase, intra-mitochondrial calcium concentration remains high even when cytosolic calcium concentration is back to rest values. The relevance of calcium uptake by mitochondria on the overall intracellular calcium homeostasis is dependent on which fraction of the calcium released from $\mathrm{SR}$ is sequestered by the mitochondria. Attempts to calculate the amount of calcium taken up by mitochondria should take into account not only the variations of $\left[\mathrm{Ca}^{2+}\right]$ mito. , but also the total volume of the mitochondria, the existence of different subpopulations of mitochondria and the impact of calcium buffers in the mitochondrial matrix.

1. Marcucci L, Canato M, Protasi F, et al. A 3D diffusionalcompartmental model of the calcium dynamics in cytosol, sarcoplasmic reticulum and mitochondria of murine skeletal muscle fibers. PLoS One 2018;13(7):e0201050. doi: 10.1371/journal.pone.0201050. eCollection 2018.

2. Scorzeto M, Giacomello M, Toniolo L, et al. Mitochondrial $\mathrm{Ca}\left({ }^{2+}\right)$-Handling in Fast Skeletal Muscle Fibers from Wild Type and Calsequestrin-Null Mice. PLoS One 2013;8(10):e74919. PubMed PMID: 24098358.

$* * * * *$

\section{Session IV - Face \& Voice Rejuvenation}

\section{Long Term home-based Surface Electrostimulation is useful to prevent atrophy in denervated Facial Muscles}

Gerd Fabian Volk 1, Dirk Arnold 2, Jovanna Thielker 1, Anne Ruck 1, Oliver Mothes 1,3, Valeria

Mastryukova 1, Winfried Mayr 4, Orlando GuntinasLichius 1
1 ENT-Department and Facial Nerve Center Jena, Jena University Hospital, Jena, Germany; 2 Institute of

Systematic Zoology and Evolutionary Biology with Phyletic Museum, Friedrich-Schiller-University Jena, Jena, Germany; 3 Computer Vision Group, FriedrichSchiller-University Jena, 4 Medical University Vienna, Center for Medical Physics and Biomedical Engineering, General Hospital Vienna, Vienna, Austria

\section{E-mail:fabian.volk@med.uni-jena.de}

Often, scull base or petrosal bone tumors and surgeries cause facial nerve paralysis and consequently result in neuromuscular atrophy of facial muscles. Because of the distance between the lesion site and the denervated muscles, reinnervating axons need several months to reach the muscles. To reduce or even prevent the muscle atrophy, electrostimulation (ES) could have a beneficial effect for facial muscles, ${ }^{1,2}$ similar to limb muscles. ${ }^{3}$ The aim of the present study is to evaluate the effects of transcutaneous facial muscle stimulation on the denervated M. Zygomaticus. As far as January 2019, we enrolled 5 patients (4 female) with facial paralysis (duration between 1 month and 16 years). The affected hemiface was the right in 3 and the left in 2 cases. The etiology of the facial paralysis, confirmed by needle Electromyography (EMG), was in all cases iatrogenic, i.e. followed benign schwannoma removal. Before the first ES, and every 4 weeks during the ES, all patients underwent regular needle EMG, ultrasound, ${ }^{4} \mathrm{MRI}^{5}$ and 3D-video. The stimulation parameters and patients home-stimulation diaries are recorded. Additionally, all patients completed the forms for Facial Disability Index (FDI) and the Facial clinometric evaluation scale (FaCE). The amplitude required to elicit a selective $\mathrm{M}$. zygomaticus contraction on the ailing hemiface was investigated at the following pulse durations: 1000, 500, $250,100,50,25,15,10,5$ and $1 \mathrm{~ms}$ both with triangular and rectangular waveform. Burst frequency was constantly kept $1 \mathrm{~Hz}$. The stimulation was performed every four weeks with the STMISOLA; BIOPAC Systems Inc. Home-training (3x5min twice a day) was individually programmed on the Stimulette r2x (Dr. Schuhfried GmbH, Vienna) for each patient. The stimulation was given in charge-balanced biphasic triangular impulses with fix build-up time (5s); build-up pause (1s); and training duration of $5 \mathrm{~min}$. Two surface electrodes $(60 \times 40 \mathrm{~mm}$ Flextrode Plus; Krauth + Timmermann) were placed as closed as possible to the lip corner on the ailing hemiface. The most effective combination of pulse duration and intensity was chosen based on the results obtained with the STMISOLA and the visual evaluation of the patient's response. Once the parameters were selected and programmed, the patient was asked to perform $5 \mathrm{~min}$ stimulation in order to exclude potential muscle fatigue. No patient reported relevant adverse events linked to home-based-training. Training with optimized electrode positioning associated with stable and specific M. zygomaticus activation, accompanied by a reduction of the necessary minimum pulse duration from 250 to $70 \mathrm{~ms}$ per phase within 16 weeks. Even before reinnervation, objective (3D-videos, sonography, MRI) and subjective (FDI, FaCE) 


\section{Sp PMD Collection of the Abstracts}

Eur J Transl Myol 29 (1):46-78 2019

parameters improved significantly compared to the prestimulation situation. ES home-based training is beneficial for patients with denervated facial muscles in reducing muscle atrophy, maintaining muscle function and improving facial symmetry. The accurate selection of the stimulation parameters and electrode position is necessary to ensure the training specifically for the selected facial muscle. A lack of relevant adverse events shows that such ES is safe. The patients showed excellent compliance with the protocol and rated the stimulation

1. Gittins J, Martin K, Sheldrick J, et al. Electrical stimulation as a therapeutic option to improve eyelid function in chronic facial nerve disorders. Investig Ophthalmol Vis Sci 1999;40: 547-54.

2. Fargher AK, Coulson SE. Effectiveness of electrical stimulation for rehabilitation of facial nerve paralysis. Physical Therapy Reviews 2017 doi: 10.1080/10833196. 2017.1368967.

3. Mayr W, Hofer C, Bijak M, Rafolt D, et al. Functional Electrical Stimulation (FES) of Denervated Muscles: Existing and Prospective Technological Solutions. Eur J Transl Myol 12, 2002;1:1-4.

4. Volk GF, Sauer M, Pohlmann M, Guntinas-Lichius O. Reference values for dynamic facial muscle ultrasonography in adults Muscle Nerve 2014;50:348-57. doi: 10.1002/mus.24204.

5. Volk GF, Karamyan I, Klingner CM, et al.. Quantitative magnetic resonance imaging volumetry of facial muscles in healthy patients with facial palsy" Plast Reconstr Surg Glob Open 2014;2:e173.

$$
* * * * *
$$

Surface Electrostimulation of Facial Muscles: Tolerability of triangular and rectangular pulses of different lengths in subjects and patients with facial palsy

Jovanna Thielker 1, Martin Christian Möller 1, Daniela Majcher 1, Valeria Mastryukova 1, Carolin Altmann 1,2, Orlando Guntinas-Lichius 1, Gerd Fabian Volk 1

1 ENT-Department and Facial Nerve Center Jena; 2 Anatomy Dept., Jena University Hospital, Germany

\section{E-mail:fabian.volk@med.uni-jena.de}

Even being used since more than 60 years, ${ }^{1}$ one often mentioned obstacle to use electrostimulation (ES) in the facial paralysis therapy is the low tolerance of the face to electricity. However, there is a lack of systematic data in support of or against such a theory. ${ }^{2-4}$ It is the aim of our study to evaluate the potential drawbacks observed when ES is applied by means of triangular pulses (TP) and rectangular pulses (RP) of different lengths to different portions of the face. We performed ES with TP and RP in 48 (58\% male, median age: 45 years) healthy subjects in the area of the eye (E), cheek (C) and mouth (M) in increasing phase duration $(0.1-500 \mathrm{~ms})$ and amplitude (0.01-40mA), detecting the motor and discomfort thresholds. In another study, 30 subjects $(73 \%$ male, median age: 49 years) and 31 patients with facial palsy and synkinesis (29\% male, median age: 52 years) were stimulated with RP and the discomfort threshold was detected. When RP ES was applied, lower amplitude than with TP ES was required to obtain a visually detectable contraction of the target muscle $(\mathrm{E} \mathrm{p}=0.01, \mathrm{C} \mathrm{p}=0.003$, $\mathrm{M} p=0.005)$. The discomfort threshold was typically reached at higher amplitudes with TP rather than with RP ES (E p $=0.03, \mathrm{C} \mathrm{p}=0.04, \mathrm{M} \mathrm{p}=0.02)$. In $84 \%$ of the cases where TP ES was applied, the amplitude required to trigger an observable response of the target muscle was above the discomfort threshold of the subject. The same observation was made only in $4 \%$ of the cases where RP ES was applied. The discomfort threshold was found significantly higher $(p=0.003)$ for TP than for RP ES when the stimulation target was the mouth corner. A lower discomfort threshold was observed when RP rather than TP ES was applied in facial palsy patients when the stimulation target was the peri-eye zone $(p=0.04)$ and the cheek $(p=0.02)$. RP seems to be better tolerated than TP ES both by healthy subjects and facial palsy patients. In general, the discomfort threshold was higher in the former than in the latter group. In all cases, the discomfort threshold was strictly depended on the individual adaptation to the stimulation.

1. Mosforth J, Taverner D. Physiotherapy for Bell's palsy. Br Med J 1958;2(5097):675-7.

2. Quinn R, Cramp F. The efficacy of electrotherapy for Bell's palsy: a systematic review. Phys Ther Rev 2003;8:151-64.

3. Teixeira LJ, Valbuza JS, Prado GF. Physical therapy for Bell's palsy (idiopathic facial paralysis). Cochrane Database Syst Rev. 2011;12:CD006283. doi: 10.1002/14651858.CD006283.pub.

4. Fargher KA, Coulson SE. Effectiveness of electrical stimulation for rehabilitation of facial nerve paralysis. Physical Therapy Reviews, DOI: 10.1080/10833196. 2017.1368967, 2017.

Functional electrical stimulation for ageing laryngeal muscles: Results from a prospective randomized human trial

Markus Gugatschka 1, Jonathan C Jarvis 2, Winfried Mayr 3, M Feiner 1, Andrijana Kirsch 1, Claus Gerstenberger 1

1. Dept of Phoniatrics, Medical University Graz, Austria; 2. School of Sport and Exercise Science, Liverpool John Moores University, United Kingdom; 3. Department of Veterinary Clinical Sciences, Royal Veterinary College, North Mymms, UK

\section{E-mail: markus.gugatschka@medunigraz.at}

The percentage of elderly people is increasing steadily during the last decades in most Western societies and large parts of Asia which is accompanied by a steady increase of age related diseases. These changes do not spare the larynx. Weakening of the voice has long been 


\section{Sp PMD Collection of the Abstracts}

Eur J Transl Myol 29 (1):46-78 2019

neglected, but gained consideration more recently, as vocal endurance is required in many professions up to higher ages. A large retrospective study identified muscular related vocal fold (VF) atrophy as the most prevalent finding. The noticeable glottal gap and VF bowing are the most prominent video-laryngoscopic findings in these patients and are related to the atrophy of the thyroarytenoid muscle (TAM). In a recent animal trial we could demonstrate that functional electrical stimulation (FES) of the afferent nerves with specific parameters led to a muscle fiber hypertrophy. ${ }^{1}$ In subsequent trials we explored not only different stimulation patterns, but also different anatomical locations and outcome parameters such as 3Dvolumtery. $^{2}$ Here we report the results of a first randomized prospective trial in humans. In a pre-trial, suitable stimulation parameters for eliciting a VF reaction were established. 14 female volunteers (mean age 66 , SD 4,6) were included, one arm received full training for eight weeks, the other arm subthreshold stimulation for four weeks, followed by four weeks of training similar to the first arm. This was followed by a wash-out phase of 6 weeks in both groups. Outcome measures comprised voice related as well as pulmonary related parameters, as well as quality of life measures. The home-based exercises comprised 3 circles of 3 minutes training, carried out five times a week. Parameters were measured at seven different time points. We could not identify a significant increase of certain voice or pulmonary parameters over the course of time in neither group. Interestingly voice related quality of life increased over the course of the trial in both groups. To the best of our knowledge this was the first prospective randomized controlled trial dealing with surface FES in aged women. We could not identify significant improvements in voice or pulmonary related parameters.

1. Karbiener M, Jarvis JC, Perkins JD, et al. Reversing Age Related Changes of the Laryngeal Muscles by Chronic Electrostimulation of the Recurrent Laryngeal Nerve. PLoS One 2016 Nov 28;11(11):e0167367.

2. Gerstenberger C, Döllinger M, Kniesburges $S$, et al. Phonation Analysis Combined with 3D Reconstruction of the Thyroarytenoid Muscle in Aged Ovine Ex Vivo Larynx Models. J Voice 2017 Sep 27. pii: S0892-1997(17)30215-1 $* * * * *$

A fast de-staining protocol after Lugol's iodinecontrasted micro-CT scan of laryngeal muscle tissue

\section{Andrijana Kirsch 1, Claus Gerstenberger 1, Justin D Perkins 2, Markus Gugatschka 1}

1 Department of Phoniatrics, Medical University of Graz, Austria; 2. Department of Veterinary Clinical Sciences, Royal Veterinary College, North Mymms, United Kingdom

Email: andrijana.kirsch@medunigraz.at

The vocal fold is housed in the larynx and is supported by a multi-facetted muscle (thyroarytenoid muscle, or TAM). We performed contrast-enhanced microcomputer tomography (micro-CT) scans of ovine larynges. Following this the TAM was reconstructed three-dimensionally using a specific software in order to define the muscle borders and to measure the volume. ${ }^{1}$ To confirm the findings of the micro-CT scans, we performed histological analysis of the very same TAM, which would allow for measurement of muscle fibre number and diameter by means of automated muscle fibre-analysis. As the samples were kept in Lugols's iodine contrast solution, we established a fast (4-day) destaining protocol of the TAM tissue that would allow subsequent histological stains. After the removal of iodine, the samples were embedded in paraffin, sectioned $5 \mu \mathrm{m}$ thick and stained using H\&E and Picrosirius red. The cross-sections of the whole ovine TAMs were scanned with Aperio Digital Pathology Slide Scanner and the resulting images were further processed by Slide $\mathbf{J}$ software, ${ }^{2}$ followed by analysis with a muscle fibreanalysing software. The quality and resolution of the obtained final images were appropriate for muscle fibreanalysing software. Unfortunately, the software could not accurately analyse the muscle images, due to tissue damage from prolonged exposure to the contrast solution. Histological analysis of muscle cross-sections is feasible after Lugol's iodine-contrasted micro-CT scan. It is however of the essence to minimize contrast exposure and to proceed to the de-staining procedure shortly after the completion of the micro-CT-scan in order to prevent tissue damage.

1. Gugatschka M, Jarvis JC, Perkins JD, et al. Functional Electrical Stimulation Leads to Increased Volume of the Aged Thyroarytenoid Muscle. Laryngoscope 2018;128:2852-7. doi: 10.1002/ lary.27342. Epub 2018 Oct 3.

2. Della Mea V, Baroni GL, Pilutti D, et al: An ImageJ plugin for automated processing of whole slide images. PLoSOne 2017;12:e0180540. doi: 10.1371 /journal.pone.0180540.

$* * * * *$

\section{Evidence that shortening of stimulus duration can alter the stimulation threshold of neuron types in peripheral nerves}

Christoph Kast 1,2, José L. Vargas Luna 1, Christian Hofer 2,3, Oskar C. Aszmann 2, Winfried Mayr 1,2

1 Center for Medical Physics and Biomedical Engineering, Medical University of Vienna, Austria; 2 Christian Doppler Laboratory for Restoration of Extremity Function, Division of Plastic and Reconstructive Surgery, Department of Surgery, Medical University of Vienna, Austria; 3 Otto Bock Healthcare Products GmbH, Vienna, Austria.

\section{Email:winfried.mayr@meduniwien.ac.at}

In a physiologically intact upper extremity, sensory perception provides haptic information, like on an object surface and grip force, and proprioception gives permanent information on the position of all arm- and hand segments in space, even without support by visual feedback. Within a wider project on upper extremity reconstruction after amputation, we work on inducing similar impressions artificially to persons with amputation, helping to integrate mechanical prosthesis 
into the body schema and facilitating acceptance and handling of artificial limbs. It has been shown, that electrical stimulation can not only reduce phantom pain in a stump after amputation but can also be applied for evoking sensory feedback. Here, we present observations on eliciting basic anatomical perception in phantom limbs by means of electrical stimulation. A self-adhesive reference electrode is applied on top of the shoulder of the amputated arm. The active counter electrode is a ballshaped metal electrode that is placed in the armpit. To enhance the electrical conductivity, electrode gel is applied. The patient has his arm stump positioned on a cushioned pedestal, lifted to $90^{\circ}$ in relation to the vertical body axis. Voltage controlled bipolar rectangular pulses with a phase width of $10 \mu$ s or $100 \mu$ s were applied in a continuous train of $1 \mathrm{~Hz}$ or $80 \mathrm{~Hz}$. At specific electrode locations and $80 \mathrm{~Hz}$ stimulation, the patients report a feeling of a "pressure sensation" in specific areas of the missing limb, or a feeling similar to having the arm "outstretched" in the 3-dimensional space, or even selective perception of single phantom fingers. Single pulse stimulation $(1 \mathrm{~Hz})$ with very short stimuli were able to induce stimulus threshold perception first as proximal sensation (distal proprioception threshold), and then with increased stimulus intensity as local skin sensation in the contact area of the active electrode (local sensory threshold); in intact individuals this test turned out to be difficult, as the motor threshold for muscle twitch reactions in the distal arm got quite strong before the local cutaneous threshold was reached. With longer pulses, e.g. $100 \mu \mathrm{s}$, the threshold amplitudes for distal proprioception and local sensory perception got more similar and selective stimulation for distal perception got difficult. Preliminary experiments have shown, that it is possible to provide a kind of proprioceptive feedback from a phantom limb of amputees while avoiding unpleasant skin sensation in the application area of the stimulation electrodes. This effect was observed when applying very short stimulation pulses and disappeared with increasing pulse duration, at least in our specific test conditions. Further work will address if and to what extent this can be useful for advancements in prosthesis control or for reducing discomfort limitations in other applications of electrical stimulation. Due to the noninvasive approach, this could be a practicable way of restoring near natural proprioceptive feedback or to reduce sensible discomfort in FES applications.

$$
* * * * *
$$

\section{Session V - Muscle Imaging}

\section{New Views of Muscle Components}

Clara Franzini-Armstrong, Manuela Lavorato

Dept of Cell and Developmental Biology, University of Pennsylvania School of Medicine, Philadelphia PA. USA

\section{E-mail:armstroc@mail.med.upenn.edu}

We have implemented a technique originally developed by T. Ogata and Y. Yamasaki and published in 1990

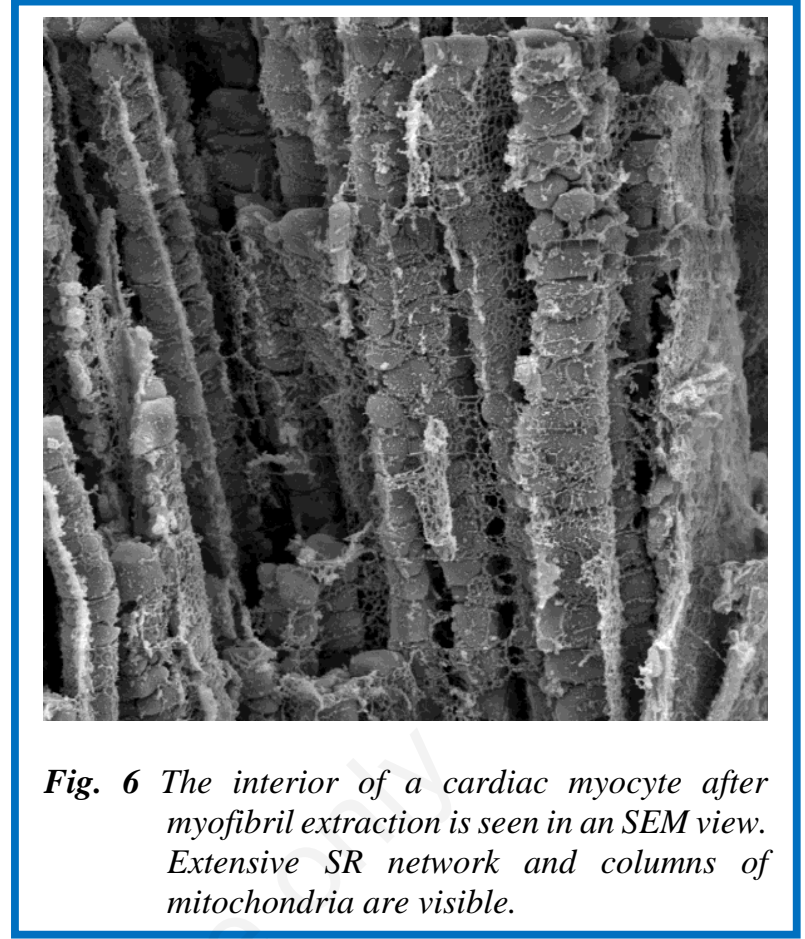

(Anat Rec.228:277-287). ${ }^{1}$ Using a diluted solution of $\mathrm{OsO} 4$ as a mordant, the protein components of tissue initially fixed in glutaraldehyde and cryofractured are solubilized and extracted, while all membrane structures are well preserved and maintained. The resulting tissue blocks are imaged in a scanning electron microscope. We have focused on two ultrastructural details. First we have examined the relationship between transverse (T) tubules and the sarcoplasmic reticulum (SR) at triads. Traditionally $\mathrm{T}$ tubules form intermittent junctions with the SR, so that triads are discontinuous in a manner that relates to the type of fiber. However we discovered that in some fish muscles triads are continuous. The functional implications of this minor but important finding will be discussed. Secondly, we took advantage of the 3-D SEM images to visualised the extent and frequency of the nanotunnel extensions that allow mitochondria in cardiac muscle to exchange matrix components (Figure 6). Cardiac but not skeletal muscle mitochondria take advantage of this unique structure.

1. Ogata T, Yamasaki Y. High-resolution scanning electron microscopic studies on the three-dimensional structure of the transverse-axial tubular system, sarcoplasmic reticulum and intercalated disc of the rat myocardium. Anat Rec 1990 Nov;228(3):277-87.

$$
* * * * *
$$

\section{Store Operated $\mathrm{Ca}^{2+}$ entry in skeletal muscle: where?}

\section{Feliciano Protasi}

CeSI-MeT, Center for Research on Ageing and Translational Medicine, University G. d'Annunzio, Chieti, Italy

E-mail:fprotasi@unich.it 


\section{Sp PMD Collection of the Abstracts}

Eur J Transl Myol 29 (1):46-78 2019

Store-operated $\mathrm{Ca}^{2+}$ entry (SOCE) is a ubiquitous cellular $\mathrm{Ca}^{2+}$ influx mechanism, first described in non-excitable cells, that is triggered by depletion of intracellular $\mathrm{Ca}^{2+}$ stores (endoplasmic reticulum, ER). A major breakthrough in the field was the identification of the two essential molecular players in SOCE: STIM1, the $\mathrm{Ca}^{2+}$ sensor in the ER, and Orai1, a $\mathrm{Ca}^{2+}$ permeable channel in the plasma membrane. ${ }^{1,2}$ SOCE is also well-documented in skeletal muscle ${ }^{4}$ where it limits muscle fatigue during repetitive fatiguing stimulation. ${ }^{3}$ Also in muscle SOCE is mediated by interactions between STIM1 in the SR and Orail channels in the PM. ${ }^{4}$ However, the precise subcellular location of STIM1-Orai1 SOCE complexes in skeletal muscle is still debated. We discovered that exercise in mice drives formation of new junctions between stacks of sarcoplasmic reticulum (SR) cisternae and transverse-tubules (TTs) containing STIM1 and Orai1, two proteins that mediate store-operated $\mathrm{Ca}^{2+}$ entry (SOCE) ${ }^{5}$. Muscles containing the new SR-TT junctions are more resistant to fatigue during highfrequency stimulation in presence of extracellular $\mathrm{Ca}^{2+}$, while this increased fatigue resistance is abolished in conditions in which $\mathrm{Ca}^{2+}$ entry in prevented (i.e. $0 \mathrm{Ca}^{2+}$ or presence of SOCE inhibitors). ${ }^{5}$ We proposed that these previously unidentified SR-TT junctions function as $\mathrm{Ca}^{2+}$ Entry Units (CEUs), providing a preferential pathway for rapid reuptake of $\mathrm{Ca}^{2+}$ into the SR during repetitive muscle activity. ${ }^{6}$ Using electron microscopy and two different functional assays we studied muscles from mice subjected to an incremental treadmill running and sacrificed within $1 \mathrm{hr}$ or after 6-24hrs. Data collected indicates that: a) while the number of SR-stacks increased up to at least $6 \mathrm{hrs}$ to return to control values only after $24 \mathrm{hrs}$ of recovery, the extension of TTs (significantly increased at $1 \mathrm{hr}$ ), returned to control values already after $6 \mathrm{hrs}$; b) fatigue resistance of EDL muscles during high-frequency stimulation and $\mathrm{Mn}^{2+}$ quench of Fura-2 fluorescence in FDB fibers were both increased after $1 \mathrm{hr}$, but were not different from control after 6 and 24hrs of recovery. Structural plasticity of TTs drives their association with SR-stack to form Calcium Entry Units (CEUs), structures providing a preferential pathway for rapid reuptake of $\mathrm{Ca}^{2+}$ into the SR during repetitive muscle activity. Our work represents a pioneer study that identified exercise-driven dynamic formation of new intracellular structures, a mechanism potentially quite important for the delay of muscle fatigue. As altered SOCE activity contributes to muscle dysfunction in ageing and various myopathies, our findings may also have implications for the understanding of mechanisms involved in muscular dysfunction.

1. Roos J, DiGregorio PJ, Yeromin AV,et al. STIM1, an essential and conserved component of store-operated $\mathrm{Ca}^{2+}$ channel function. J Cell Biol 2005;169:435-45. Epub 2005 May 2.

2. Vig M, Peinelt C, Beck A et al. CRACMI is a plasma membrane protein essential for store-operated $\mathrm{Ca}^{2+}$ entry. Science 2006;26;312(5777):1220-3. Epub 2006 Apr 27.

3. Lyfenko AD, Dirksen RT. Differential dependence of storeoperated and excitation-coupled $\mathrm{Ca}^{2+}$ entry in skeletal muscle on STIM1 and Orai1. J Physiol 2008;586:4815-24.
4. Wei-Lapierre L, Carrell EM, Boncompagni S, et al. Oraildependent calcium entry promotes skeletal muscle growth and limits fatigue. Nat Commun 4, 2805 (2013).

5. Boncompagni S, Michelucci A, Pietrangelo L., et al. Exercise-dependent formation of new junctions that promote STIM1-Orail assembly in skeletal muscle. Sci Rep 2017;7:14286.

$$
* * * * *
$$

\section{Exercise prevents formation of Tubular Aggregates in ageing skeletal muscle fibers}

\section{Simona Boncompagni}

\author{
CeSI-MeT, Center for Research on Ageing and \\ Translational Medicine, University G. d'Annunzio, \\ Chieti, Italy \\ Email: s.boncompagni@unich.it
}

Tubular aggregates (TAs), unusual accumulation of sarcoplasmic reticulum (SR) tubes in skeletal muscle fibers, ${ }^{1}$ have been recently linked to mutations in STIM1 and Orai $1,{ }^{2,3}$ the two main molecular players in storeoperated $\mathrm{Ca}^{2+}$ entry (SOCE). ${ }^{4}$ SOCE is a ubiquitous mechanism that is activated by depletion of intracellular stores that allows recovery of extracellular $\mathrm{Ca}^{2+}$ during repetitive muscle activity. We have previously shown that: i) TAs also form in muscle of male mice during ageing starting from the remodeling of SR tubes/vesicles at the I -band ${ }^{5}$ and ii) exercise triggers the formation of unique intracellular junctions between SR and TTs (named $\mathrm{Ca}^{2+}$ Entry Units, or CEUs) ${ }^{6}$ that promotes colocalization of Orai1 with STIM1 and improves muscle function in presence of external $\mathrm{Ca}^{2+}$. We used a combination of electron and confocal microscopy (EM and $\mathrm{CM}$ ), western blotting (WB), and functional studies (ex-vivo stimulation protocols in presence of absence of external $\mathrm{Ca}^{2+}$ ) to compare ultra-structure, STIM1-Orai1 localization / expression, and fatigue resistance of EDL muscles dissected from adult and aged mice, the latter divided in 2 groups: control and exercised in wheel cages for 15 months. The results collected in the structural experiments (CM and EM) indicated that: i) ageing causes STIM1 and Orai1 to accumulate in TAs; and ii) exercise significantly reduced formation of TAs. Parallel functional studies revealed that iii) aged EDL exhibit a faster decay of contractile force than adult muscles, likely caused by their inability to recruit extracellular $\mathrm{Ca}^{2+}$; and iv) exercise restored the lost capability to use external $\mathrm{Ca}^{2+}$ of aged EDL muscles by promoting maintenance/rescue of CEUs. Our results suggest that exercise prevents improper accumulation of STIM1 and Orai1 in TAs during ageing restoring the capability of aged muscle to use external $\mathrm{Ca}^{2+}$ via SOCE.

1. Chevessier F, Marty I, Paturneau-Jouas M, et al. Tubular aggregates are from whole sarcoplasmic reticulum origin: alterations in calcium binding protein expression in mouse skeletal muscle during ageing. Neuromuscul Disord 2004; 14:208-16.

2. Böhm J, Chevessier F, Maues De Paula A, et al. Constitutive activation of the calcium sensor STIM1 causes tubular-aggregate myopathy. Am J Hum Genet 2013; 92:271-8. 
3. Bohm J, Bulla M, Urquhart JE, et al. ORAII Mutations with Distinct Channel Gating Defects in Tubular Aggregate Myopathy. Hum Mutat 2017;38:426-38.

4. Michelucci A, García-Castañeda M, Boncompagni S, Dirksen RT. Role of STIM1/ORAI1-mediated storeoperated Ca2+ entry in skeletal muscle physiology and disease. Cell Calcium 2018;76:101-15.

5. Boncompagni $S$, Protasi $F$ and Franzini-Armstrong $C$. Sequential stages in the age-dependent gradual formation and accumulation of tubular aggregates in fast twitch muscle fibers: SERCA and calsequestrin involvement. Age (Dordr). 2012;34:27-41.

6. Boncompagni S, Michelucci A, Pietrangelo L, et al. Addendum: Exercise-dependent formation of new junctions that promote STIM1-Orail assembly in skeletal muscle. Sci Rep 2018;8(1):17463.

$$
* * * * *
$$

\section{Structure and Function Magnetic Resonance Imaging (MRI) to Study Muscle Atrophy in Uni- Lateral Limb Suspension (ULLS)}

\section{Shantanu Sinha}

Dept. of Radiology, Muscle Imaging and Modeling Lab,

\section{University of California at San Diego, School of Medicine, San Diego, CA, USA \\ Email:shsinha@ucsd.edu}

MRI, by virtue of its excellent soft-tissue contrast, nonionizing and non-invasive nature, has become one of the most powerful radiologic imaging modality for in-vivo human studies. This talk will start with an overview of the technical aspects of MRI that yield unique multiparametric mapping of the MSK system. We will first present studies of static properties such as muscle structure, including shape and size (using high-resolution MRI), microarchitecture and fiber architecture (from Diffusion Tensor Imaging) and tissue composition (e.g. adipose tissue, fibro-cartilaginous extracellular matrix using Ultrashort-TE (UTE) imaging) (Figure 7). We will next focus on Dynamic (using Velocity-Encoded PhaseContrast (VE-PC)) MR imaging which maps muscle tissue (velocity and strain) while executing different motion paradigms such as passive, isometric, concentric or eccentric, using a computer-controlled, MR compatible system. Novel MRI methods to decrease scan time based on compressed sensing will be discussed; these methods will expand the applicability of MR dynamic imaging to higher maximum voluntary contractions in shorter times, allowing "weak" (e.g. senior) subjects to be studied. Such combined studies allow a comprehensive assessment of normal and diseased muscles, tendon and the extracellular matrix. The ULLS model has been established as a reference method to induce controlled atrophy, allowing the study of effects of unloading without other confounding effects of disease. Post-suspension changes in indices such as voxel based strain-rate and strain-tensor, extracted from the velocity maps, will be discussed. Our study reveal that the correlation of these indices to force loss with suspension enables one to identify potential contributions to loss of muscle quality from modulation of the extracellular matrix with unloading. We will conclude

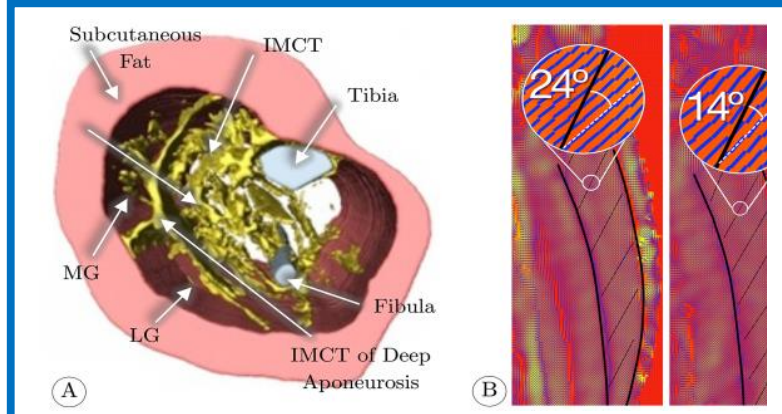

Fig. 7 A, First ever 3D visualization (yellow) of the Intramuscular Connective Tissue network, acquired with innovative UTE imaging. $\boldsymbol{B}$, Principal component of the Strain rate tensor (SRT). Inset shows angle in Young (240, left) and $\operatorname{Old}(140$, right), between fiber direction (black) and Principal component of SRT (blue). Larger angles may imply larger contributions from lateral compared to longitudinal force transmission (along muscle fiber direction).

with our future/ongoing work including further development of the MR techniques and analysis, validation of structural changes measured by MR with muscle biopsy histological analysis, and validation of the conclusions of dynamic MRI by computational modeling.

1. Csapo $R$, Malis $V$, Sinha $U$, et al. Age-associated differences in triceps surae muscle composition and strength - an MRI-based cross-sectional comparison of contractile, adipose and connective tissue. BMC Musculoskelet Disord 2014;15:209.

2. Sinha $S$, Sinha $U$, Edgerton VR. In vivo diffusion tensor imaging of the human calf muscle. J Magn Reson Imaging 2006;24(1):182-90.

3. Sinha S, Hodgson JA, Finni T, et al. Muscle kinematics during isometric contraction: Development of phase contrast and spin tag techniques to study healthy and atrophied muscles. J Magn Reson Imaging 2004;20:100819.

4. Lustig M, Donoho D, Pauly JM. Sparse MRI: The application of compressed sensing for rapid MR imaging. Magn Reson Med 2007;58:1182-95.

$$
* * * * *
$$

\section{Structure and Function Magnetic Resonance Imaging (MRI) to Study Muscle Atrophy in Uni-Lateral Limb Suspension (ULLS) \\ Usha Sinha \\ Department of Physics, University of California at San Diego, San Diego, CA, USA Email: usinha@ucsd.edu}

The soft tissue contrast afforded by Magnetic Resonance Imaging makes it an ideal candidate for imaging the musculoskeletal system. Recent advances in structural MRI enable quantitative voxel based estimation of fat fraction and fibrosis in muscle. Further, diffusion tensor 


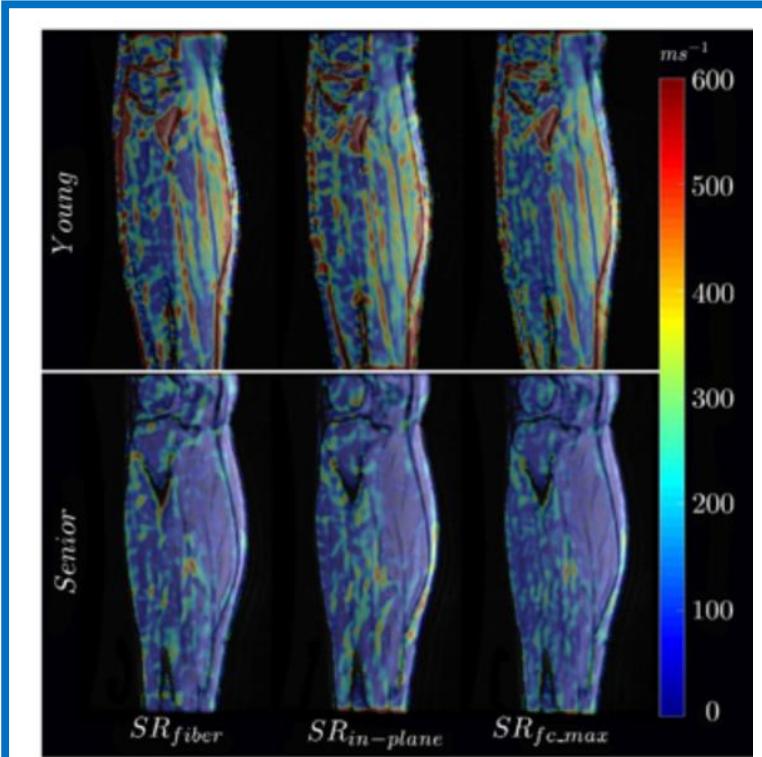

Fig. 8 Strain rate maps of the normal strain rates (SRfiber, SRin-plane) and shear strain rates (SR fc-max) in a young and a senior subject at the same force level. The maps show lower values in both the normal and shear strain rates in the senior subject.

imaging provides a unique method to look at muscle fiber architecture as well the microstructure of the fiber/ endomysium. Extraction of microstructural parameters from gross MR indices requires a biophysical model linking fiber/ endomysium structure and permeability to the indices derived from diffusion tensor imaging ${ }^{1}$. Another tool to study macromolecular fraction (e.g., collagen) is magnetization transfer contrast that allows an indirect way of looking at the macromolecules in muscle ${ }^{2}$. The talk will start with the overview of the novel structural MRI techniques including extension of conventional diffusion tensor imaging techniques to enable modeling to extract microstructural parameters. As functional MR imaging has been discussed at length in this workshop, the focus for functional imaging in this talk will be on the image analysis to extract the strain rate tensors and their physical implications (Figure 8). The concept of shear strain will be introduced; the difference in this index between the young and old cohorts will be discussed. We extend the hypothesis that the shear strain may reflect the remodeling of the ECM and these findings are discussed in the context of structural changes in the aging muscle ${ }^{3,4}$. The talk will conclude with a discussion of ongoing work on biophysical diffusion models to extract fiber microstructural parameters as well as mapping the macromolecular fraction in muscle to elucidate structural changes that may provide insights into loss of muscle strength with aging. ${ }^{5}$

1. Winters $K V$, Reynaud O, Novikov DS, et al. Quantifying myofiber integrity using diffusion MRI and random permeable barrier modeling in skeletal muscle growth and Duchenne muscular dystrophy model in mice. Magn Reson
Med 2018;80:2094-2108. doi: 10.1002/mrm.27188. Epub 2018 Mar 25.

2. Li K, Dortch RD, Kroop SF, et al. A rapid approach for quantitative magnetization transfer imaging in thigh muscles using the pulsed saturation method. 2015;33:70917. doi: 10.1016/j.mri.2015.03.003. Epub 2015 Apr 1.

3. Sinha U, Malis V, Csapo R, et al. Age-related differences in strain rate tensor of the medial gastrocnemius muscle during passive plantarflexion and active isometric contraction using velocity encoded MR imaging: potential index of lateral force transmission. Magn Reson Med 2015;73:1852-63. doi: 10.1002/mrm.25312. Epub 2014 Jul 8.

4. Sinha $U$, Malis $V$, Csapo $R$, et a. Shear strain rate from phase contrast velocity encoded MRI: Application to study effects of aging in the medial gastrocnemius muscle.J Magn Reson Imaging 2018;48:1351-7. doi: 10.1002/jmri.26030. Epub 2018 Apr 1.

5. Malis V, Sinha U, Csapo R, et al. Diffusion tensor imaging and diffusion modeling: Application to monitoring changes in the medial gastrocnemius in disuse atrophy induced by unilateral limb suspension.J Magn Reson Imaging 2018 Dec 19. doi: 10.1002/jmri.26295. [Epub ahead of print].

$* * * * *$

Human muscle fascicle behavior during drop jumping in conditions of normal, hypo- and hypergravity

Marco Narici 1, Monti E 1; Ritzmann R 3; Freyler $K$ 3; Albracht K 4,5; Waldvogel, J 3; Helm M 3; Staudle B 4; Cesare N 2; Pavan P 2; Gollhofer A 3

1 Institute of Physiology, Department of Biomedical Sciences, University of Padova, Italy; 2 Department of Industrial Engeneer, University of Padova, Padova, Italy; 3 Department of Sport and Sport Science, University of Freiburg, Freiburg, Germany; 4 Institute of Biomechanics and Orthopaedics, German Sport

University Cologne, Cologne, Germany; 5 Department of Medical Engineering and Technomathematics, Aachen University of Applied Sciences, Aachen, Germany

\section{Email:marco.narici@unipd.it}

In preparation of landing during a drop jump performed on Earth, the central nervous system (CNS) must preactivate the lower limb muscles to stiffen the joints and generate a muscle contraction able to break the fall upon impact with the ground. During the landing phase (from ground contact until minimum ankle joint) the CNS must fix the muscle length in order to allow the tendon to store elastic energy to be used during the push off phase. In the push off phase, the muscle shortens, and the tendon releases the elastic energy stored during the landing phase (Figure 9). However, in conditions of gravity different from that on Earth, the strategies operated by the neuromuscular system to enable safe landing and efficient jumping, are presently unknown. Hence the aims of the present study were to determine, 1) how is 


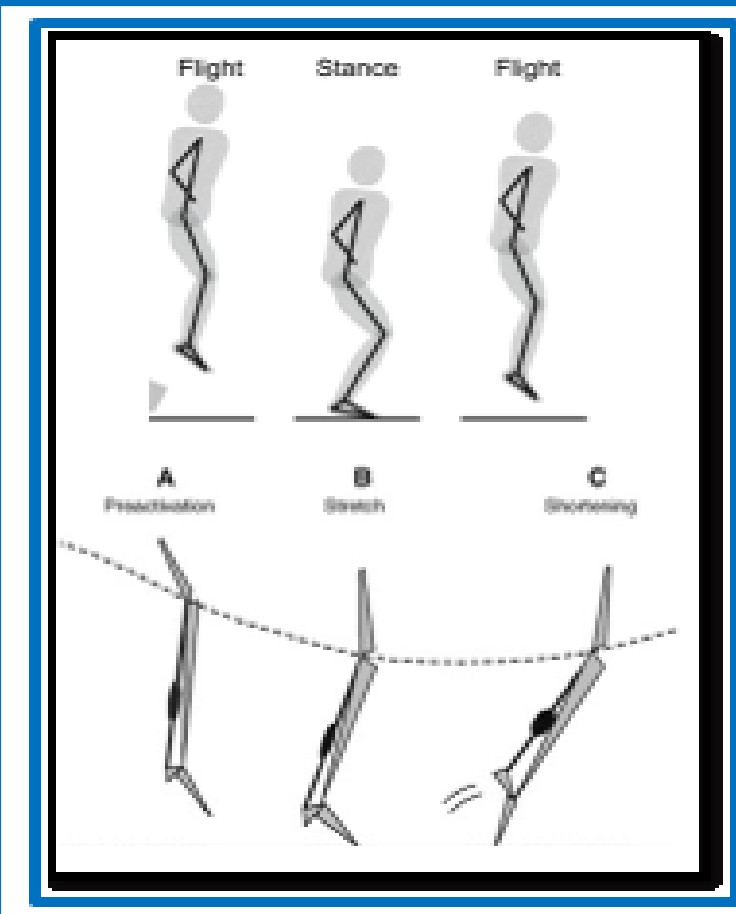

Fig. 9 During landing phase the CNS must fix the muscle length in order to allow the tendon to store elastic energy to be used during the push off phase.

muscle fascicle length regulated in preparation for landing and jumping in variable g levels, and 2) in what portion of the sarcomere length-tension (L-T) relation are fascicles operating during drop jumping in variable $g$ levels? In order to answer these questions, measurements of gastrocnemius medialis muscle fascicle length (by ultrasound), electromyographic activity and ground reaction force (by force platform) were obtained in 18 male volunteers in repeated drop jumps (DJ) performed at $0.25,0.50,0.75,1.0,1.25,1.5$ and $2.0 \mathrm{~g}$ during 30 parabolas of a parabolic flight (Fig.2). When the time course of changes in fascicle length during the four phases of the DJ (i-pre-activation, ii-ground contact, iiimaximum ankle joint, iv-push off phase), it was found that both in hypo-g $(0.25,0.50,0.75 \mathrm{~g})$ and hyper-g $(1.25$, 1.5 and $2.0 \mathrm{~g}$ ), muscle pre-activation occurred earlier than in $1.0 \mathrm{~g}$. In all $\mathrm{g}$ conditions, during the pre-activation phase, fascicles shorted to about $60 \%$ of their initial length after which fascicles behaved quasi-isometrically up the push off phase. However, in hypo-g the quasiisometric phase was reached about $100 \mathrm{~ms}$ later than in $1 \mathrm{~g}$. In hyper-g the quasi-isometric phase was much shorter than in $1 \mathrm{~g}$, as time was needed to recover from the eccentric phase following ground-contact. Examination of the gastrocnemius L-T relation, assuming an optimum sarcomere length of $2.75 \mu$ (Walker and Schrodt, 1973), ${ }^{1}$ revealed that, in all conditions, sarcomeres operated in the ascending limb of the L-T relation- However, whereas in $1 \mathrm{~g}$ at the end of pre-activation sarcomeres were close to optimum length $(2.75 \mu)$, in both hypo-g and hyper-g sarcomere length was about $2.0 \mu$. Remarkably, in both hypo-g and hyper$\mathrm{g}$, at the start of the push-off phase, sarcomere length was 1.3-1.5 $\mu$. In conclusion, the motor system is able to put in place effective strategies for safely landing in hypo and hyper-g and it does so by achieving an early preactivation in both conditions (Figure 10). Pre-activation is greater in hyper-g since muscle loading already occurs as the subject is standing on the step before the jump. However, regardless the $\mathrm{g}$ levels, fascicles are still able to achieve the essential quasi isometric phase to enable

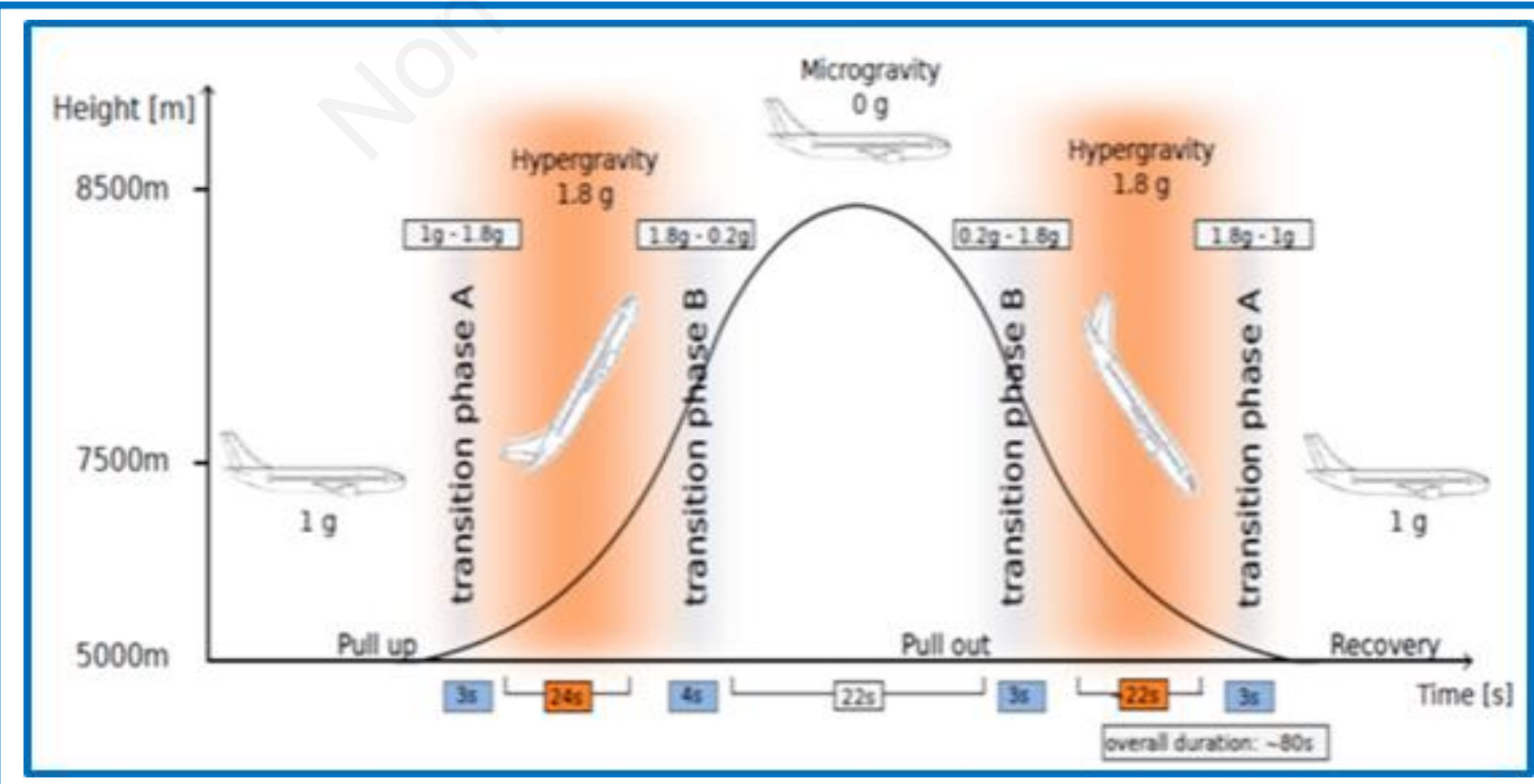

Fig. 10 The typical profile of a flight parabola. 
tendon stretch, storage and release of elastic energy for the following jump.

1. Walker SM, Schrodt GR. I segment lengths and thin filament periods in skeletal muscle fibers of the Rhesus monkey and the human. Anat Rec. 1974 Jan;178(1):63-81.

$* * * * *$

\section{In-vivo ultrasound assessment of biceps femoris: architecture heterogeneity and region-specific changes in Shear Wave Elastography modulus}

\section{Martino V Franchi 1, 2, Fitze DP 1, Raiteri BJ 3, Hahn D 3, Spörri J 1}

1 Sports Medical Research Group \& Laboratory for Muscle Plasticity, Department of Orthopaedics, Balgrist University Hospital, University of Zurich, Switzerland; 2 Institute of Physiology, Department of Biomedical Sciences, University of Padua, Italy; 3 Ruhr-University of Bochum, Department of Sports Science, Bochum, Germany

\section{Email: martino.franchi@unipd.it}

Biceps Femoris long head (BFlh) has lately been in the research spotlight. Adaptations in muscle architecture, and especially in fascicle length (Lf), have been investigated, in response to training and in relation to injury prevention mechanisms. Lf is commonly assessed by ultrasonography and estimated by single snapshots taken at specific region of interests (ROIs). As single snapshots are dependent on the field of view of the transducer (commonly $4-5 \mathrm{~cm}$ length), the visible portion of the fascicles results being $~ 50 \%$ of the total Lf, leaving half of the length to be extrapolated. BFlh presents a heterogeneous architecture, and extrapolation methods may represent a problem. Extended field of view ultrasound (EFOV) technique may be advantageous by providing a panoramic view of the whole fascicles. Moreover, the non-uniform anatomical features of BFlh could result in distinct regional changes in visco-elastic properties (measured by Shear Wave Elastography) in response to acute or chronic exercise. Right BFlh muscle architecture (Lf, pennation angle -PA and muscle thickness -MT) of 37 elite athletes $(21.7 \pm 2.8 \mathrm{y}$, mean \pm SD) was assessed. Ultrasound snapshots were acquired at $50 \%$ of femur length between using a $5 \mathrm{~cm}$ linear transducer. EFOV scans were acquired from the distal to proximal insertions. Shear Wave modulus was calculated after 6 maximal repetitions of Nordic Hamstring Exercise at distal $(30 \%$ femur length, $0=$ mid patellar point) and proximal (70\% femur length) sites during a passive shortening and subsequent lengthening of BFlh (knee passive flexion from 0 to $\sim 70$ degrees ROM). There were significant differences in muscle architecture of BFlh between MID and Distal sites (Lf mid $=8.07 \pm 1.36 \mathrm{~cm}$ vs. Lf distal $=7.65 \pm 1.27 \mathrm{~cm}$, $\mathrm{P}<0.05)$. Shear Wave Elastography showed differences between changes at different muscle sites, with the distal portion resulting in higher change in Shear Wave Modulus. The present results showed the heterogeneity of BFlh muscle architecture and of its acute response to exercise. This raises a call for caution for physiologists, biomechanics and sports medicine experts investigating adaptations to training, muscle models, and injury prevention strategies. Further technique developments (i.e., DTI, EFOV) should be implemented in order to understand this "compartmentalisation" of responses in structural and mechanical properties of BFlh.

$$
* * * * *
$$

\section{Ultrasound and electromyographic assessment of the multifidus spinae muscle: Reliability, function and relevance for sarcopenia}

Elena Monti, Brogi M 2, Tagliaferri S 3,4, Ticinesi A 3,4, Maggio M 3,4, Narici MV 1

1 Department of Biomedical Sciences, University of Padova, Italy; 2 School of Sports Medicine, Department of Medicine, University of Padova, Italy

3 Department of Clinical and Experimental Medicine, University of Parma, Parma, Italy

4 Geriatric-Rehabilitation Department, Parma University Hospital, Parma, Italy

Email: elena.monti.1@phd.unipd.it

The multifidus spinae (MF) is an essential muscle for preserving an erect posture and for rotating the spine. This muscle undergoes significant atrophy with prolonged bed rest' and ageing. ${ }^{2}$ The aims of the present study were to, 1) quantify the activity of the MF muscle during basic activities of daily living (ADL), 2) evaluate the reliability of ultrasound measures of MF crosssectional area (CSA) and, 3) establish the degree of MF sarcopenia in community-dwelling independent older individuals. Nine young female controls (aged 20-35 years) and 9 older females (aged $70 \geq$ years), diagnosed as sarcopenic according to the EWGSOP definition, ${ }^{3}$ were tested in this study. MF CSA was measured at L5 by ultrasound on both sides of the spine using a $3-11 \mathrm{MHz}$ linear probe. Electromyographic (EMG) activity of the MF during the 5- repetitions chair stand test (CST) and the $4 \mathrm{~m}$ gait speed test (GST) was recorded using surface electrodes. Activity of the MF, normalised to its maximum voluntary contraction EMG, was $45 \%$ during the CST and $21 \%$ during the GST. ICC of MF CSA measurements at L-5 was good to excellent (0.8910.998). MF CSA of the older participants was found to be $29.3 \%$ smaller of the younger controls $(p<0.01)$. The multifidus spinae muscle of community-dwelling independent septuagenarian females was found to be significantly sarcopenic (ca. 30\% smaller CSA than of young controls). Given the considerable activation of the MF during basic ADLs, sarcopenia of this muscle, particularly when exacerbated by disuse, may seriously impact on mobility and independence of older people. Sarcopenia of the MF muscle may be reliably assessed by ultrasound imaging

1. Belavý DL, Armbrecht G, Richardson CA, Felsenberg D, Hides JA. Muscle atrophy and changes in spinal morphology: is the lumbar spine vulnerable after prolonged bed-rest? Spine (Phila Pa 1976). 2011 Jan 15;36(2):137-45.

2. Cuellar WA, Wilson A, Blizzard CL, Otahal P, Callisaya ML, Jones G, Hides JA, Winzenberg TM. The assessment of abdominal and multifidus muscles and their role in physical 
function in older adults: a systematic review. Physiotherapy. 2017 Mar;103(1):21-39.

3. Cruz-Jentoft A, Bayens JP, Bauer JM, et al. Sarcopenia: European consensus on definition and diagnosis. Age Ageing. 2010;39:412-23.

$$
* * * * *
$$

Dynamic Echo Imaging of FES muscle activation and perfusion in SCI

\section{Ugo Carraro}

1 A\&C M-C Foundation for Translational Myology, Padova, Italy; 2 Department of Biomedical Sciences, University of Padova, Italy

Email:ugo.carraro@unipd.it

Denervation of a defined skeletal muscle is due to lower motor neuron (LMN) or peripheral nerve lesions that have major consequences on the muscle tissue. After early atrophy, the mid- and late-phases presents two very contrasting myofibers populations: beside those severely atrophic with internalized groups of myonuclei, large fast-type muscle fibers continue to be present 4 to 6 years after Spinal Cord Injury (SCI). Recent results of rat experiments provides the rational basis for understanding the residual functional characteristics of the long-term denervated muscle and the molecular explanation of its ability to respond to home-base functional electrical stimulation (h-b FES) using custom-designed electrodes and stimulators. Further outcomes of the Vienna-Padova ten-year collaboration are: 1. a world-unique Myo- Bank of muscle biopsies and 2. improved imaging procedures (Color Computer Tomography (CT) scan and Functional Echomyography), all demonstrating that h-b FES induces improvements in muscle contractility, tissue composition and mass, despite permanent LMN denervation. The benefits of h-b FES could be extended from patients suffering with complete Conus-Cauda Syndrome to the numerous patients with incomplete LMN denervation of skeletal muscles to determine whether h-b FES reduces secondary complications related to disuse and impaired blood perfusion (reduction in bone density, risk of bone fracture, decubitus ulcers, and pulmonary thromboembolism). We are confident that translation of the results of a clinical experiment, from the EU Project RISE to the larger cohort of incomplete LMN denervated muscles will provide the wanted results.

1. Kern H, Stramare $R$, Martino L, et al. Permanent LMN denervation of human skeletal muscle and recovery by $h-b$ FES: management and monitoring. Eur J Transl Myol, 2010;20:91104. https://doi.org/10.4081/ejtm.2010.1806.

2. Zanato R, Martino L, Stramare R. Functional Echo myography of the human denervated muscle: first results. Eur J Transl Myol:2011;21:3-30.

3. Zanato $R$, Martino $L$, Carraro $U$, et al. Functional Echomyography: thickness, ecogenicity, contraction and perfusion of the LMN denervated human muscle before and during h-bFES. Eur J Transl Myol 2010;20:33-40.

4. Zanato R, Stramare R, Boato N, et al. Dynamic Echomyography Shows That FES in Peripheral Denervation does not Hamper Muscle Reinnervation. Biomed Tech (Berl). 2013 Aug;58 Suppl 1. pii: /j/bmte.2013.58.issue-s1-A/bmt-2013-4034/bmt-20134034.xml. doi: 10.1515/bmt-2013-4034. Epub 2013 Sep 7.
5. Ortolan P, Zanato R, Coran A, et al .Role of Radiologic Imaging in Genetic and Acquired Neuromuscular Disorders. Eur J Transl Myol. 2015 Mar 11;25(2):5014. doi:

10.4081/ejtm.2015.5014. eCollection 2015 Mar 11. Review.

$* * * * *$

\section{March 30, 2019 Hotel Augustus, Euganei Hills (Padova), Italy}

\section{Official Meeting of the EU Center of Active Aging (CAA)}

\section{Official Meeting of the EU Center of Active Aging: Introduction, Helmut Kern}

Physiko und Rheumatherapie - Institut für Physikalische Medizin, St. Pölten, Austria

Email:helmut@kern-reha.at

\section{Senior fitness program in Bratislava: age related training, assessments \\ Jan Cvecka \\ Bratislava, Slovakia}

Email: cvecka@fsport.uniba.sk

$* * * * *$

Literature overview in seniors: training methods, outcome, cost effectiveness

Milan Sedliak Veronika Tirpakova,

Bratislava, Slovakia,

Email:msedliak@yahoo.com

$$
* * * * *
$$

\section{EU Center of Active Aging (CCA)}

\section{CAA Project overview}

\section{Stefan Loefler}

(1) Physiko und Rheumatherapie - Institut für Physikalische Medizin, St. Pölten, Austria; (2) Ludwig Boltzmann Institute for Rehabilitation Research, St. Pölten, Austria

Email: stefan.loefler@kern-reha.at

Physical inactivity is a global pandemic that not only causes morbidity and mortality, but also represents a major economic burden worldwide. As a longer-term goal, we must strive to integrate physical activity into our everyday lives. ${ }^{1}$ Increased physical activity, among other things, has an influence on chronic pain of the locomotor system, minimizes the risk of cardiovascular disease, increases self-esteem and cognitive abilities, maintains mobility and autonomy, and thus has direct effects on healthy life years and direct and indirect health 
expenditure. The CAA project is intended to be in line with the Austrian health targets (R-GZ) for Austria, ${ }^{2}$ the National Health Program of Slovakia, ${ }^{3}$ the recommendations of the Lancet Physical Activity Series Executive Committee, ${ }^{4}$ and $\mathrm{WHO}^{5}$ encouraging policymakers to take physical activity seriously, to motivate people, and to create the opportunities to do so on a regular basis. Over the entire project period of three years, more than 1000 people aged 60 years and over will benefit from the offers of the CAAs in the program area. Within the scope of the project, they should carry out a standardized training (10 weeks) followed by home training for 12 months.

1. Andersen LB, Mota J, Di Pietro L. Update on the global pandemic of physical inactivity. Lancet 2016;388(10051):1255-6.

2. Health Targets Austria. Austrian Federal Ministry of Health and Women's Affairs. 2012

3. National Health Promotion Programme (Slovakia) Update [Internet]. Ministry of Health of the Slovak Republic; 2014

4. Das P, Horton R. Physical activity - time to take it seriously and regularly. Lancet. 2016 Sep 24;388(10051):1254-5.e.

5. Physical activity strategy for the WHO European Region 2016-2025 [Internet]. WHO 2016.

$* * * * *$

\section{New trainings and assessments of CAA} Dusan Hamar

Bratislava, Slovakia,

Email: hamar@fsport.uniba.sk $* * * * *$

Physical Conditioning Interventions in Older Adults: Literature Review with the Focus on Training Program Description, Study Design and Statistical Analysis

Nejc Sarabon

University of Primorska, Faculty of Health Sciences, Izola, Slovenia

\section{Email: Nejc.Sarabon@fvz.upr.si}

The principle of progression is a fundamental rule in physical conditioning and includes modulation of exercise intensity, volume, frequency and content. Optimal exercise plan provides an individual with sufficient load to elicit adaptations (i.e. improvement in physical abilities) whilst allowing sufficient rest to prevent injuries or overtraining (Lorenz and Morrison, 2015). ${ }^{1}$ As the proportion of elderly people in population is growing, assessing the effects of different interventions to counteract ageing and prevent diseases is of paramount importance (Kirk-Sanchez and McGough, 2014). ${ }^{2}$ We conducted a systematic search of the scientific literature to explore the extent to which exercise interventions for elderly were designed and described. The majority of the studies did not progress the difficulty of the exercises or did not clearly specify whether progression was included. Exercise interventions were often combined with nutritional interventions (Denison et al.), ${ }^{3}$ cognitive training or both (Ngandu et al., 2015). ${ }^{4}$ Content, volume and frequency of the exercise was described sufficiently in most studies, while more care should be given to description exercise intensity. Future research should explore the effects of more carefully designed interventions based on the principle of progressive overload.

1. Lorenz D, Morrison S. Current concepts in periodization of strength and conditioning for the sports physical therapist. International journal of sports physical therapy 2015;10:734

2. Kirk-Sanchez NJ, McGough EL. Physical exercise and cognitive performance in the elderly: current perspectives. Clinical interventions in aging 2014;9:51.

3. Denison HJ, Cooper C, Sayer AA, Robinson SM. Prevention and optimal management of sarcopenia: a review of combined exercise and nutrition interventions to improve muscle outcomes in older people. Clinical interventions in aging 2015;10:859.

4. Ngandu T, Lehtisalo J, Solomon A, et al. A 2 year multidomain intervention of diet, exercise, cognitive training, and vascular risk monitoring versus control to prevent cognitive decline in at-risk elderly people (FINGER): a randomised controlled trial. The Lancet 2015;385(9984), 2255-63.

$$
* * * * *
$$

\section{Neurophysiological aspects of CAA}

\section{Sascha Sajer, Felix Kabas}

Vienna, Austria,

Email: office@drsajer.at

$$
* * * * *
$$

\section{Insights into muscle atrophy and sarcopenia: mechanisms and countermeasures}

\section{Antonio Musarò}

\section{Sapienza University of Rome, Italy}

Email: antonio.musaro@uniromal.it

The decline in functional performance and restriction of adaptability represents the hallmark of sarcopenia. The characteristic loss in muscle mass, coupled with a decrease in strength and force output, has been associated with a selective activation of catabolic pathways, a general reduction in survival mechanisms, and alteration in the repair mechanisms. When homeostatic mechanisms are blunted, as in the elderly, muscle tends to be replaced by non-contractile fibro-adipose tissue, with ensuing loss of muscle function, a phenomenon that poses an outstanding social burden, as the aged population increases. Understanding the molecular mechanisms of sarcopenia and how satellite cell pool is preserved and cells efficiently recruited to maintain muscle mass and function is a key issue in tackling this problem. We will discuss the signalling pathways involved in sarcopenia and the cellular and molecular mechanisms that modulate satellite cell activity and muscle regeneration. 


\section{Sp PMD Collection of the Abstracts}

Eur J Transl Myol 29 (1):46-78 2019

Exercise induces structural remodeling of intracellular organelles deputed to $\mathrm{Ca}^{2+}$ handling and aerobic ATP production in muscle of mice and human seniors

\section{Feliciano Protasi}

CeSI-MeT, Center for Research on Ageing and Translational Medicine, University G. d'Annunzio, Chieti, Italy

\section{E-mail:fprotasi@unich.it}

Proper muscle function is controlled by intracellular $\mathrm{Ca}^{2+}$ concentration, which in turn depends on: a) release of $\mathrm{Ca}^{2+}$ from intracellular stores during excitation contraction (EC) coupling, which activates muscle contraction; b) entry of $\mathrm{Ca}^{2+}$ from the extracellular space via store-operated $\mathrm{Ca}^{2+}$ entry (SOCE), a mechanism important to limit muscle fatigue; c) uptake of $\mathrm{Ca} 2+$ into the mitochondrial matrix, which stimulates aerobic ATP production; and finally d) sequestration/removal by sarcoplasmic reticulum (SR) and plasma membrane (PM) pumps, which relaxes muscle fibers. Abnormalities in $\mathrm{Ca}^{2+}$ handling underlies many physiopathological conditions. For instance, reduced SR Ca2+ release has been linked to fatigue and dysfunction in ageing, while excess of SR Ca2+ leak may even underlie life-threatening conditions such as malignant hyperthermia susceptibility (MHS). In the last 10-12 years we have collected compelling evidence that the proper architecture and function of all those membrane systems involved in $\mathrm{Ca}^{2+}$ handling and aerobic ATP production depends on muscle activity: a. denervation causes disarray of units deputed to EC coupling (calcium release units, CRUs) and mitochondrial apparatuses, while functional electrical stimulation (FES) promotes some rescue of this structural disarray; ${ }^{1}$ b. sedentary ageing in mice and humans causes misplacement of mitochondria and partial disarray of CRUs, while longterm training does prevent effectively those changes; ${ }^{2-4}$ c. acute exercise promotes functional assembly of SOCEsites (calcium entry units, CRUs), while post-exercise recovery determines their disassemble ${ }^{6} \mathrm{~d}$. sedentary ageing causes accumulation of dysfunctional proteins in tubular aggregates (TAs), while exercise prevents TAs formation (manuscript in preparation). $\mathrm{Ca}^{2+}$ handling is crucial for muscle function and is controlled by diverse membrane systems and intracellular organelles. Our experience collected in different, but complementary, projects: a) indicates the structure and function of intracellular organelles is preserved or rescued by exercise or training; and b) underlines the importance of physical exercise during ageing to preserve muscle function.

1. Boncompagni S, Kern H, Rossini K, et al. Structural differentiation of skeletal muscle fibers in the absence of innervation in humans. Proc Natl Acad Sci U S A. 2007 104(49):19339-44.

2. Pietrangelo L, D'Incecco A, Ainbinder A, et al. Agedependent uncoupling of mitochondria from $\mathrm{Ca}^{+}$release units in skeletal muscle. Oncotarget 2015;6:35358-71.
3. Zampieri S, Pietrangelo L, Loefler S, et al. Lifelong physical exercise delays age-associated skeletal muscle decline. J Gerontol A Biol Sci Med Sci 2015;70:163-73.

4. Pietrangelo L, Michelucci A, Ambrogini P, et al 2018. Muscle activity prevents the uncoupling of mitochondria from Ca2+ Release Units induced by ageing and disuse. Arch Biochem Biophys 2019;663:22-33. doi: 10.1016/j.abb.2018.12.017. Epub 2018 Dec 20.

5. Boncompagni $S$, Michelucci A, Pietrangelo L, et al. Exercise-dependent formation of new junctions that promote STIM1-Orail assembly in skeletal muscle. Sci Rep2017;7:14286.

$$
* * * * *
$$

\section{Home-based Functional Electrical Stimulation recovers epidermis from atrophy and flattening induced by Spinal Cord Injury}

Giovanna Albertin 1, Barbara Ravara 2,3, Helmut Kern 4, Christian Hofer 5, Diego Guidolin 1, Andrea Porzionato 1, Anna Rambaldo 1, Raffaele De Caro 1, Sandra Zampieri 2,3,4, Amber Pond 6, Mauro Alaibac

$$
\text { 7, Ugo Carraro 2,3 }
$$

1 Department of Neuroscience, Section of Human Anatomy, University of Padova, Italy; 2

Interdepartmental Research Center of Myology, Department of Biomedical Science, University of Padova, Italy; 3 A\&C M-C Foundation for

Translational Myology, Padova, Italy; 4 Physiko- und Rheumatherapie, St. Poelten, Austria; 5 Ludwig Boltzmann Institute of Electrical Stimulation and Physical Rehabilitation, Vienna, Austria; 6 Anatomy Department, Southern Illinois University School of Medicine, Carbondale, IL, USA; 7 Department of Medicine, Section of Dermatology Clinic, University of Padova, Italy.

\section{Email: giovanna.albertin@unipd.it}

The description of skin adaptation to electrical stimulation by surface electrodes may offer new opportunities to study the skin on dermatological complications that may develop after SCI for reasons related to the injury or to the life styles of SCI patients, since the skin becomes more vulnerable to tissue hypoxia that antagonizes endogenous healing cascades. ${ }^{1}$ After a preliminary study of three patients, ${ }^{2}$ here we extend the study adding quantitative histologic and immunohistochemical analyses of skin biopsies harvested from both legs of 13 patients. HematoxilinEosin (HE) stain and immunohistochemical procedure to give morphological information about epidermis and analyze CD1a as specific marker of LCs were carried out. ${ }^{2-4}$ The HE staining revealed a regular stratified squamous epithelium rich in cells with an organization with ridges and papillae in some sample but in several the epidermis appears very atrophic, i.e., thinner and flattened, with strong evidence that epidermis progressively decreases in thickness and in dermal papillae complexity with SCI time. After two additional years of SCI, but with 5-day per week h-bFES, a significant recovery in epidermis thickness, interdigitation Index and the number of papillae-like structures per mm of skin was shown. The skin biopsies 
with an anti-epidermal CD1a antibody immunostaining shown that LCs were present in all biopsies, but they were not significantly increased or decreased with SCI timing and/ or h-bFES. This skin information about use of the electrical stimulation could be an important contributory mechanism to prevent and/or manage skin disorders, in particular pressure sores in SCI, metabolic disease and aging sarcopenia.

1. Marbourg JM, Bratasz A, Mo X, Popovich PG. Spinal Cord Injury Suppresses Cutaneous Inflammation: Implications for Peripheral Wound Healing. Neurotrauma. 2017;34:1149-55. doi: 10.1089/neu.2016.4611. Epub 2016 Oct 17.

2. Albertin $G$, Hofer $C$, Zampieri S, et al. In complete SCI patients, long-term functional electrical stimulation of permanent denervated muscles increases epidermis thickness. Neurol Res 2018;40:277-82. doi: 10.1080/0161 6412.2018.1436877. Epub 2018 Feb 15.

3. Ravara B, Hofer C, Kern H, et al. Dermal papillae flattening of thigh skin in Conus Cauda Syndrome. Eur J Transl Myol 2018;28(4):7914. doi: 10.4081/ejtm. 2018.7914. eCollection 2018 Nov 2.

4. Albertin G, Kern H, Hofer C, et al. Two years of Functional Electrical Stimulation by large surface electrodes for denervated muscles improve skin epidermis in SCI. Eur J Transl Myol 2018;28(1):7373. doi: 10.4081/ejtm.2018. 7373. eCollection 2018 Jan 12.

$* * * * *$

\section{Early Rehabilitation after knee and hip replacement}

\section{Early Rehabilitation Project Overview}

\section{Helmut Kern 1,2}

1 Physiko und Rheumatherapie - Institut für Physikalische Medizin, St. Pölten, Austria; 2 Ludwig Boltzmann Institute for Rehabilitation Research, St. Pölten, Austria

Email: helmut@kern-reha.at

In comparison to other European countries, Austria is one of the leaders in hip and knee replacement with 273 and 216 patients per 100000 inhabitants, respectively. ${ }^{1}$ In recent years, both surgical techniques and postoperative rehabilitation have made great progress, but in Austria waiting times for inpatient rehabilitation of up to 8 weeks are not uncommon and outpatient rehabilitation is not yet established nationwide. Our hypothesis is that early start of post-operative rehabilitation is essential for rapid restoration of joint function, reduction of pain, and earlier occupational and social participation. The aim of the project is to compare the existing scheme of a combination of hospitalization and inpatient rehabilitation with the new approaches. The focus here is the treatment with underwater gymnastics and underwater pressure jet massage supplemented by physical pain therapy, therapeutic gymnastics and medical training therapy. The basis for the project is the cooperation with the Lower Austrian Healthcare and Social Fund (NÖGUS) and their associated orthopedic and trauma surgery departments of several university hospitals.

1. EUROSTAT (2016). Surgical operations and procedures performed in hospitals by ICD-9-CM. [hlth_co_proc2]

\section{$* * * * *$}

\section{Literature review of rehabilitation methods after knee/hip TEP}

Christian Hofer

LBI Electrical Stimulation, Vienna, Austria

Email: christian.hofer@rehabilitationresearch.eu

$$
* * * * *
$$

\section{Sweat and saliva analyses of Myokines in training} and rehabilitation

Barbara Ravara 1,2, Sandra Zampieri 1,2,3, Giulio Sauro Guardiero 2,4, Ugo Carraro 1,2

1 Interdepartmental Research Centre of Myology (CIRMyo), Department of Biomedical Sciences, University of Padova, Italy; 2 A\&C M-C Foundation for

Translational Myology, Padova, Italy; 3 Physiko- und Rheumatherapie, St. Poelten, Austria; 4 Palestra Padova, Italy

\section{Email: barbara.ravara@unipd.it}

Sweat is the secretion product of the skin sweat glands, that are located at forehead, scalp, armpits, palm of hands and sole of feet. When secretion of sweat (perspiratio insensibilis) is not compensated by full evaporation, drops of liquid accumulate on the skin surface. Sweat is a colorless hypotonic liquid, whose composition includes electrolytes, mainly sodium, potassium, magnesium and chlorides, lactic acid metabolites, creatinine, and glucose, but many other molecules, including peptides and proteins can by identified and quantitated by micro-, nano- or pico-methods. In case of inflammation and/or infection, anti-microbial peptides (defensins) and proinflammatory cytokines, including interleukins and tumor necrosis factors, are identified. When body temperature rises, sweat secretion increases with the consequent loss of liquids. If not compensated by an adequate intake of water dehydration occurs, a frequent complication observed in elderly people even at normal temperature conditions. Various types of sweating are described: i) thermal sweating may occur as a response to body exposure to increased environmental temperature, limitation of perspiration insensibilis by impermeable layer of clothes or to increased load of metabolic calories induced by physical work; ii), psychic or emotional sweating due to particular moods; iii) pharmacological sweating due to chemical and pharmacological components. ${ }^{1}$ Among physiological conditions, exercise-induced sweat lactate levels and their relationship to physiological parameters such as gender, age and physical fitness, along with the effect of climate adaptation on sweat lactate concentrations are well described in many reviews. ${ }^{2}$ Among pathological conditions, sweat analysis is the standard method for the 


\section{Sp PMD Collection of the Abstracts}

Eur J Trans1 Myol 29 (1):46-78 2019

diagnosis of cystic fibrosis. ${ }^{3,4}$ The analysis is based on the quantitative determination of the chlorides ions in the perspiration liquid induced by skin pilocarpine iontophoresis that stimulates production of visible sweat. ${ }^{5}$ If inflammation of the skin occurs, as in the case of atopic dermatitis, as a result of damage to the protective barrier of the epidermis cytokines and chemokines come into contact with keratinocytes and stimulate their activation. These keratinocytes activated by inflammatory cytokines increase the signal pathway expression of NF-KB, ERK, JNK proteins. ${ }^{6}$ In physiological conditions, skin epithelial cells produce a basal level of cytokines and chemokines through sweat. Therefore, cytokines and chemokines represent sound biomarkers that may be found also in sweat. Proinflammatory cytokines are routinely quantitated by immune-enzymatic tests, which require the use of colorimetric ELISA kits. Our aim is to extend these analyses to the MYOKINES, that is cytokines secreted by muscle cells or acting on muscle cells. A feasibility study will be performed in youngs comparing the concentration of MYOKINES in the sweat induced by increased body temperature either limiting sweats evaporation in resting people or increasing sweat production by physical exercise. A set of commercially available MYOKINES, that is, the interleukins IL- $1 \alpha$, IL$1 \beta$, IL-6, IL-8, IL-31 will be measured using commercially available ELISA Kits. ${ }^{7-12}$ The sweat will be collected with filter paper, from forehead and armpits. The collected sweat will be centrifuged at $15,000 \mathrm{rpm}$ for 10 min for sediment cell debris while the supernatant will be frozen in liquid nitrogen, aliquoted and stored at $-80^{\circ}$ $\mathrm{C}$ until ELISA analyses. The total protein concentration of the sweat supernatant will be quantified by the Bradford method. The concentration of cytokines in the sweat in the literature is expressed as $\mathrm{ng} / \mathrm{mg}$ of total sweat proteins. ${ }^{13}$ Since it is difficult to calculate how much sweat is collected from time to time, we proposed to monitor the chemokyne content using as a reference the content of sweat glycoproteins in an unknown amount of sweat. Thus, sweat supernatant will be also subjected to protein electrophoresis in order to separate its proteins. ${ }^{14}$ The protein components of sweat that can be identified are usually: pre-albumin, albumin, "fast" alpha2, "slow" alpha2, gamma-globulin and alpha 1 and alpha 2 globulin that are absent in serum. This electrophoresis approach to identify sweat proteins is a very useful method for quantifying protein components present in small quantities such as the glycoproteins and has proved useful in clinical study of mucoviscidosis, a disease associated with abnormalities of sweat secretion. ${ }^{15}$ Furthermore, extending the analyses to saliva will open new analytical tools to follow up training in young and elderly persons and in rehabilitation strategies based on volitional or ES-induced exercise.

1. Mena-Bravo A, Luque de Castro MD. Sweat: a sample with limited applications and promising future in metabolomics. J Pharm Biomed Anal. 2014 Mar;90:139-47. doi: 10.1016/j.jpba.2013.10.048. Epub 2013 Dec 10. Review.

2. Derbyshire PJ1, Barr H, Davis F, Higson SP. Lactate in human sweat: A critical review of research to the present day. J Physiol Sci 2012;62:429-40. doi: 10.1007/s12576012-0213-z. Epub 2012 Jun 8.

3. Luque de Castro MD. Sweat as a clinical sample: what is done and what shoud be done. Bioanalysis 2016;8, 85-8.

4. Marques-Deak A, Cizza G, Eskandari $F$, et al. Measurament of cytokines in sweat patches and plasma in healthy women: validation in a controlled study. J Immunol Methods 2006;315:99-109.

5. Gibson LE, Cooke RE. A test for concentration of electrolytes in sweat in cystic fibrosis of pancreas utilizing pilocarpine by iontophpresis. Pedriatics 1958;24:545-9.

6. Dai X, Okazaki H, Hanakawa $M$, et al. Eccrine sweat contains IL1- $\alpha, I L-1 \beta$ and IL-31 and activates epidermal Keratinocytes as a danger signal. PLos ONE 2013;8(7):e67666.

7. Jones AP, Webb LM, Anderson AO, et al. Normal human sweat contains interleuchin-8. J Leukoc Biol 1995;5:434-7.

8. Sato K, Sato F. Interleukin-1 alpha in human sweat is functionally active and derived from the eccrine gland. Am J Physiol 1994;266:R950-9.

9. Dambacher J, Beigel F, Seiderer J, et al. Interleukin 31 mediates MAP kinase and STAT 1/3 activation in intestinal epithelial cells and its expression is upregulated in inflammatory bowel disease. 2007;56:1257-65.

10. Reitamo S, Anttila HS, Didierjean L, Saurat JH. Immunoistochemical identification of interleukin 1 alpha and beta in human eccrine sweat-gland apparatus. $\mathrm{Br} J$ Dermatol 1990;122:315-23.

11. Cornelissen C, Luscher-Fizlaff, Baron JM, Luscher B. Signaling by IL-31 and functional consequences. Eur J Cell Biol 2012;91:552-66.

12. Dinarello CA. Inteleukin-1. Cytokine Growth Factor Rew 1997;8,253-65.

13. Pallavicini JC, Othmar G, di Sant'Agnese P, Buskirk ER. Isolation and characterization of carbohydrate-protein complexes from human sweat. Annals of the New York Academy of Sciences 1963;106:330.

14. Jirka M. Micro-Disc electrophoresis of proteins in pilocarpine-induced sweat. Febs Letters 1969;4:28-30.

15. Jirka M. An alpha2-globulina component present in sweat, saliva, tears, human milk, colostrum and cerumen. Febs Letters 1968;1:77-80.

$$
* * * * *
$$

\section{Adaptations of dermal collagen and microvessels to aging, exercise and $h$-bFES}

\section{Giovanna Albertin 1, Barbara Ravara 2,3, Andrea} Porzionato 1, Ugo Carraro 2,3, Helmut Kern 4

1 Department of Neuroscience, Section of Human Anatomy, University of Padova, Italy; 2 Interdepartmental Research Center of Myology, Department of Biomedical Science, University of Padova, Italy; 3 A\&C M-C Foundation for

Translational Myology, Padova, Italy; 4 Physiko- und Rheumatherapie, St. Poelten, Austria

\section{Email: giovanna.albertin@unipd.it}

A healthy skin barrier against dehydration, penetration of microorganisms, allergens, and other irritant agents is an 


\section{Sp PMD Collection of the Abstracts}

Eur J Transl Myol 29 (1):46-78 2019

important component of a good quality of life. Daily skin care may increase skin regeneration, elasticity, smoothness, and thus delay skin complications, while degradation of skin primary structural constituents, i.e., elastin and fibrous collagen, significantly occurs in old person and may occur in early aging, mimicking the effects of chronic sun exposure. Meantime, changes of the micro vessels accompany or precedes those changes. In literature cell proliferation and migration are described as positive responses to endogenous electrical currents or external electrical stimulation used to promote these biological processes for the treatment of ulcers and other chronic wounds. Our aims are to evaluate elastic and fibrous collagen fibers, capillaries and lymphatic vessels on skin biopsies harvested from spinal cord injury patients before and after two years of h-bFES. This information may correlate or not with the adaptations we recently described on epidermal thickness, ${ }^{1,2}$ and epidermal-dermal morphology that strongly support effectiveness of electrical stimulation in DDM SCI persons. The skin biopsies of a subset of SCI patients enrolled in the EU Project RISE will be used. All subjects suffering with conus and cauda equina syndrome were volunteers and agree to perform two years of h-bFES training and to offer skin and muscle biopsies from right and left leg, both before and after h-bFES (Ethikkommission der Stadt Wien EK-02-068-0702). ${ }^{3-4}$ Preliminary results will be reported on skin sections, stained by Verhoff-Van Gieson method. Using the image analysis using imageJ the staining property of the fibrous collagen and of elastic fibers will be processed by segmenting out of the image and appropriately adjusting the colour settings. ${ }^{5}$ The area covered will be calculated by the software in terms of number of pixel. Furthermore, we will performe immunostaining of blood microvasculature by $\mathrm{CD} 31,{ }^{6}$ and lymphatic vessels by LYVE-1 antibodies. Normal skin biopsies harvested during surgery will be stained for fibrous and elastic collagen and microvasculature to follow-up skin changes with age.

1. Albertin $G$, Hofer C, Zampieri S, et al. In complete SCI patients, long-term functional electrical stimulation of permanent denervated muscles increases epidermis thickness. Neurol Res 2018;40:277-282. doi: 10.1080/01616412.2018.1436877. Epub 2018 Feb 15.

2. Albertin G, Kern H, Hofer C, et al. Two years of Functional Electrical Stimulation by large surface electrodes for denervated muscles improve skin epidermis in SCI. Eur J Transl Myol. 2018;28(1):7373. doi: 10.4081/ejtm.2018.7373. eCollection 2018 Jan 12.

3. Kern H, Carraro U, Adami N, et al. Home-based functional electrical stimulation rescues permanently denervated muscles in paraplegic patients with complete lower motor neuron lesion. Neurorehabil Neural Repair. 2010;24:709721. DOI:10.1177/1545968310366129. Epub 2010 May 11.

4. Kern H, Carraro U. Home-based functional electrical stimulation ( $h$-b FES) for long- term denervated human muscle: history, basics, results and perspectives of the Vienna rehabilitation strategy. Eur J Transl Myol
2014;24:27-40. DOI: 10.4081/ejtm.2014.3296 eCollection 2014 Mar 31.

5. Diekmann J, Alili L, Scholz, Oet al. A Three-dimensional skin equivalent reflecting some aspects of in vivo aged skin. Exp Dermatol 2016;25:56-61. doi: 10.1111/exd.12866. Epub 2015 Nov 23.

6. Bentov I, Reed MJ. The effect of aging on the cutaneous microvasculature. Microvasc Res 2015;100:25-31. doi: 10.1016/j.mvr.2015.04.004. Epub 2015 Apr 24. Review.

$$
* * * * *
$$

\section{Muscle mass and nuclear density in atrophy, recovery from atrophy, and hypertrophy after resistance training}

\section{Mark R Viggars 1, Andrew Fisher 2, Jonathan C. Jarvis 1,2}

1 Research Institute for Sport and Exercise Sciences, Liverpool John Moores University, Liverpool, UK; 2 Department of Musculoskeletal Biology, Institute of Ageing and Chronic Disease, University of Liverpool, Liverpool, $U K$

\section{Email: J.C.Jarvis@ljmu.ac.uk}

Nuclei contain the majority of a cell's genetic material, organised into the DNA-protein complex known as a chromosome. The appropriate transcription of chromosomal genes is pivotal for cell function and control of growth in the presence or absence of mechanical and hormonal stimuli. Skeletal muscle myofibers are long, cylindrical cells. Because of the relatively large volume of cytoplasm within these myofibers, homeostasis is maintained by multiple myonuclei dispersed along the length of the sarcolemma. Myofibers can hypertrophy in response to mechanical and hormonal stimuli by increasing protein synthesis and/or decreasing protein degradation. During hypertrophy they may recruit additional myonuclei from satellite cells, to support the larger cytoplasmic volume. ${ }^{1}$ In our new co-contraction model of resistance exercise, we find that 10 days of electrical stimulation to produce resisted contractions in the tibialis anterior (TA) muscle, results in an increase in myonuclei number per fibre cross-section of $20.97 \% \pm 4.55 \% \quad(\mathrm{P}<0.05)$. During muscle atrophy, (often found as a result of ageing, cancer cachexia, and disuse) it has been suggested that 'excess' nuclei may be removed by selective apoptosis, therefore regulating myonuclear domain size such that fibres have the same myonuclear domain size in both atrophic and hypertrophic conditions. Previous studies have presented conflicting results, possibly because of the difficulty in distinguishing between myogenic nuclei and the nuclei of supporting tissues and blood vessels. ${ }^{1}$ We report that silencing the TA muscle via tetrodotoxin (TTX) administration to the common peroneal nerve, results in a significant reduction $(\mathrm{P}<0.05)$ of muscle mass of 6.9 , 29.5 and $50.7 \% .^{2}$ We now show related decreases in myonuclear domain size of $8.5,15.9$ and $30.8 \%$ after 3 , 7 and 14 days of atrophy (Fig 1). Following 7 days of habitual physical activity after 14 days of TTX administration, mass and myonuclear domain size recovered from -50.7 to $-24.5 \%$ and -30.8 to $17.1 \%$ 
respectively. Despite these large changes in mass and the area of cytoplasm for which a myonuclei supplies mRNA, there were no significant differences in myonuclei number per $10 \mu \mathrm{m}$ fibre cross-section across all conditions, measured using the novel marker pericentriolar material-1 (PCM1), which differentiates between myonuclei of the post-mitotic muscle fibres and those of supporting tissues (Winje et al., 2018) (Fig 2). Considerable atrophy and recovery from atrophy can thus occur without substantial loss or multiplication of myonuclei, in contrast with the hypertrophy caused by resistance exercise. However, we did find a significant reduction in total nuclei number with the specific DNA marker DAPI $(9.3 \%, \mathrm{P}<0.05)$ (Fig 2) after 14 days of atrophy suggesting that, since the myonuclear number did not change in those cells, nuclei of non-myogenic tissues such as stromal or vascular cells might be lost through apoptosis after a more prolonged period of atrophy.

1. Gundersen K. Muscle memory and a new cellular model for muscle atrophy and hypertrophy. J Exp Biol 2016; 219(Pt 2):235-42. doi: 10.1242/jeb.124495. Review.

2. Fisher AG, Seaborne RA, Hughes TM, et al. Transcriptomic and epigenetic regulation of disuse atrophy and the return to activity in skeletal muscle. FASEB J 2017;31:5268-82. doi: 10.1096/fj.201700089RR. Epub2017 Aug 17.

3. Winje IM, Bengtsen M, Eftest $\phi l$ E, et al.Specific labelling of myonuclei by an antibody against pericentriolar material 1 on skeletal muscle tissue sections. Acta Physiologica 2018;223:e13034. doi: 10.1111/apha.13034. Epub 2018 Feb 1.

\section{SpPMD POSTER SESSION Always on display}

\author{
Monitor Myokines of sweat and saliva in training \\ and rehabilitation: Science or Fiction? \\ Barbara Ravara 1,2, Sandra Zampieri 1,2,3, Giulio \\ Sauro Guardiero 2,4, Ugo Carraro 1,2
}

1 Interdepartmental Research Centre of Myology (CIRMyo), Department of Biomedical Sciences, University of Padova, Italy; 2 A\&C M-C Foundation for

Translational Myology, Padova, Italy; 3 Physiko- und

Rheumatherapie, St. Poelten, Austria; 4 Palestra

Padova, Italy

\section{Email: barbara.ravara@unipd.itu}

Sweat is the secretion product of the skin sweat glands, that are located at forehead, scalp, armpits, palm of hands and sole of feet. When secretion of sweat (perspiratio insensibilis) is not compensated by full evaporation, drops of liquid accumulate on the skin surface. Sweat is a colorless hypotonic liquid, whose composition includes electrolytes, mainly sodium, potassium, magnesium and chlorides, lactic acid metabolites, creatinine, and glucose, but many other molecules, including peptides and proteins can by identified and quantitated by micro-, nano- or pico-methods. In case of inflammation and/or infection, anti-microbial peptides (defensins) and proinflammatory cytokines, including interleukins and tumor necrosis factors, are identified. When body temperature rises, sweat secretion increases with the consequent loss of liquids. If not compensated by an adequate intake of water dehydration occurs, a frequent complication observed in elderly people even at normal temperature conditions. Various types of sweating are described: i) thermal sweating may occur as a response to body exposure to increased environmental temperature, limitation of perspiration insensibilis by impermeable layer of clothes or to increased load of metabolic calories induced by physical work; ii), psychic or emotional sweating due to particular moods; iii) pharmacological sweating due to chemical and pharmacological components. ${ }^{1}$ ]. Among physiological conditions, exercise-induced sweat lactate levels and their relationship to physiological parameters such as gender, age and physical fitness, along with the effect of climate adaptation on sweat lactate concentrations are well described in many reviews. ${ }^{2}$ Among pathological conditions, sweat analysis is the standard method for the diagnosis of cystic fibrosis. ${ }^{3,4}$ The analysis is based on the quantitative determination of the chlorides ions in the perspiration liquid induced by skin pilocarpine iontophoresis that stimulates production of visible sweat. ${ }^{5}$ If inflammation of the skin occurs, as in the case of atopic dermatitis, as a result of damage to the protective barrier of the epidermis cytokines and chemokines come into contact with keratinocytes and stimulate their activation. These keratinocytes activated by inflammatory cytokines increase the signal pathway expression of NF-KB, ERK, JNK proteins. ${ }^{6}$ In physiological conditions, skin epithelial cells produce a basal level of cytokines and chemokines through sweat. Therefore, cytokines and chemokines represent sound biomarkers that may be found also in sweat. Proinflammatory cytokines are routinely quantitated by immune-enzymatic tests, which require the use of colorimetric ELISA kits. Our aim is to extend these analyses to the MYOKINES, that is cytokines secreted by muscle cells or acting on muscle cells. A feasibility study will be performed in youngs comparing the concentration of MYOKINES in the sweat induced by increased body temperature either limiting sweats evaporation in resting people or increasing sweat production by physical exercise. A set of commercially available MYOKINES, that is, the interleukins IL- $1 \alpha$, IL$1 \beta$, IL-6, IL-8, IL-31 will be measured using commercially available ELISA Kits. ${ }^{7-12}$ The sweat will be collected with filter paper, from forehead and armpits. The collected sweat will be centrifuged at $15,000 \mathrm{rpm}$ for 10 min for sediment cell debris while the supernatant will be frozen in liquid nitrogen, aliquoted and stored at $-80^{\circ}$ $\mathrm{C}$ until ELISA analyses. The total protein concentration of the sweat supernatant will be quantified by the Bradford method. The concentration of cytokines in the sweat in the literature is expressed as $\mathrm{ng} / \mathrm{mg}$ of total sweat proteins. ${ }^{13}$ Since it is difficult to calculate how much sweat is collected from time to time, we proposed to monitor the chemokyne content using as a reference the content of sweat glycoproteins in an unknown amount 


\section{Sp PMD Collection of the Abstracts}

Eur J Transl Myol 29 (1):46-78 2019

of sweat. Thus, sweat supernatant will be also subjected to protein electrophoresis in order to separate its proteins. ${ }^{14}$ The protein components of sweat that can be identified are usually: pre-albumin, albumin, "fast" alpha2, "slow" alpha2, gamma-globulin and alpha 1 and alpha 2 globulin that are absent in serum. This electrophoresis approach to identify sweat proteins is a very useful method for quantifying protein components present in small quantities such as the glycoproteins and has proved useful in clinical study of mucoviscidosis, a disease associated with abnormalities of sweat secretion. ${ }^{15}$ Furthermore, extending the analyses to saliva will open new analytical tools to follow up training in young and elderly persons and in rehabilitation strategies based on volitional or ES-induced exercise.

1. Mena-Bravo A, Luque de Castro MD. Sweat: a sample with limited applications and promising future in metabolomics. J Pharm Biomed Anal. 2014 Mar;90:139-47. doi: 10.1016/j.jpba.2013.10.048. Epub 2013 Dec 10. Review.

2. Derbyshire PJ1, Barr H, Davis F, Higson SP. Lactate in human sweat: A critical review of research to the present day. J Physiol Sci 2012;62:429-40. doi: 10.1007/s12576012-0213-z. Epub 2012 Jun 8.

3. Luque de Castro MD. Sweat as a clinical sample: what is done and what shoud be done. Bioanalysis 2016;8, 85-8.

4. Marques-Deak A, Cizza G, Eskandari $F$, et al. Measurament of cytokines in sweat patches and plasma in healthy women: validation in a controlled study. J Immunol Methods 2006;315:99-109.

5. Gibson LE, Cooke RE. A test for concentration of electrolytes in sweat in cystic fibrosis of pancreas utilizing pilocarpine by iontophpresis. Pedriatics 1958;24:545-9.

6. Dai X, Okazaki H, Hanakawa M, et al. Eccrine sweat contains IL1- $\alpha, I L-1 \beta$ and IL-31 and activates epidermal Keratinocytes as a danger signal. PLos ONE 2013;8(7):e67666.

7. Jones AP, Webb LM, Anderson AO, et al. Normal human sweat contains interleuchin-8. J Leukoc Biol 1995;5:434-7.

8. Sato K, Sato F. Interleukin-1 alpha in human sweat is functionally active and derived from the eccrine gland. Am J Physiol 1994;266:R950-9.

9. Dambacher J, Beigel F, Seiderer J, et al. Interleukin 31 mediates MAP kinase and STAT 1/3 activation in intestinal epithelial cells and its expression is upregulated in inflammatory bowel disease. 2007;56:1257-65.

10. Reitamo S, Anttila HS, Didierjean L, Saurat JH. Immunoistochemical identification of interleukin 1 alpha and beta in human eccrine sweat-gland apparatus. $\mathrm{Br} J$ Dermatol 1990;122:315-23.

11. Cornelissen C, Luscher-Fizlaff, Baron JM, Luscher B. Signaling by IL-31 and functional consequences. Eur J Cell Biol 2012;91:552-66.

12. Dinarello CA. Inteleukin-1. Cytokine Growth Factor Rew 1997;8,253-65.

13. Pallavicini JC, Othmar G, di Sant'Agnese P, Buskirk ER. Isolation and characterization of carbohydrate-protein complexes from human sweat. Annals of the New York Academy of Sciences 1963;106:330.

14. Jirka M. Micro-Disc electrophoresis of proteins in pilocarpine-induced sweat. Febs Letters 1969;4:28-30.
15. Jirka M. An alpha2-globulina component present in sweat, saliva, tears, human milk, colostrum and cerumen. Febs Letters 1968; 1:77-80. $* * * * *$

\section{New animal models of sarcoglycanopathy} Martina Scano, Giovanni Risato, Michela Soardi,
Marcello Carotti, Dorianna Sandonà

Department of Biomedical Sciences, University of Padova, Padova, Italy

\section{Email: dorianna.sandona@unipd.it}

Sarcoglycans (SG) are four proteins essential for the sarcolemma integrity of striated muscles during contraction. In sarcoglycanopathies, most of the reported cases are due to missense mutations originating a full length but folding-defective proteins that cause the strong reduction of the SG-complex on the sarcolemma. ${ }^{1,2} \mathrm{We}$ already published exciting results about a novel therapeutic approach based on small-molecules able to help protein folding, leading to proper SG complex localization on sarcolemma of primary myotubes from a LGMD2-D patient. ${ }^{3}$ To confirm in vivo this strategy, we need animal models expressing folding-defective sarcoglycans. The existing SG-null mice are unsuitable to our purpose, while the KI models do not display any clinical symptom of the pathology. In light of these limitations, and considering the large number of reported sarcoglycan missense mutants, our aim is the generation and characterization of novel $\alpha$-sarcoglycanopathy models by the transduction of null mice pups with rAAVs expressing different missense mutants of the human $\alpha$ SG. Our data demonstrate the possibility to obtain a dystrophic phenotype in the presence of a mutated protein, but the setting up of quantitative methods is now necessary. On the other hand, considering the failed mouse KI models developed until now, we are also focusing on a new zebrafish model, taking advantage of CRISPR-cas9 system for the generation of both $\mathrm{KO}$ and KI models. We already obtained the generation of both delta-SG and beta-SG KO lines, for which we already started characterization. Our preliminary data shows loss of SG complex at the membrane and decreased motor activity. We are now setting up the injection of human mRNA expressing WT or mutated human protein. In the meanwhile, we are testing different CRISPR/Cas9 approaches for the generation of KI models, which will represent the model of election for a faster and reproducible drug screening.

1. Kirschner J, Lochmuller H. Sarcoglycanopathies. Handb Clin Neurol 2011;101:41-6.

2 Carotti M, Fecchio C, Sandona D. Emerging therapeutic strategies for sarcoglycanopathy. Expert Opin Orphan D 2017;5:381-96.

3. Carotti M, Marsolier J, Soardi M, et al. Repairing foldingdefective alpha-sarcoglycan mutants by CFTR correctors, a potential therapy for limb-girdle muscular dystrophy 2D. Human Molecular Genetics 2018;27:969-84. 


\section{Sp PMD Collection of the Abstracts}

Eur J Transl Myol 29 (1):46-78 2019

Orthodontic treatment on lip, masseter and tongue pressure in children

Giuseppe Messina 1,2,3, Valerio Giustino 4, Luca

Petrigna 4, Nemania Lakicevic 4, Fabio Scoppa 5,

Alessio Pirino 6, Angelo Iovane 1,2, Antonino Bianco

1,7, Antonio Palma.1,7,8.

1; Department of Psychology, Educational Science and

Human Movement, University of Palermo, Palermo,

Italy 2; Posturology and Biomechanics Laboratory

Research Unit, University of Palermo, Palermo, Italy; 3

Posturalab Italia Research Institute, Palermo, Italy; 4

PhD Program in Health Promotion and Cognitive

Sciences, University of Palermo, Palermo, Italy; 5

Faculty of Medicine and Dental Surgery, Sapienza

University of Rome, Rome, Italy; 6 Department of

Biomedical Sciences, Division of Human Anatomy,

University of Sassari, Sassari, Italy; 7 Sport and

Exercise Sciences Research Unit, University of

Palermo, Palermo, Italy; 8 Regional Sport School of

Sicily CONI (Olympic National Italian Committee), Palermo, Italy

\section{Email: giuseppe.messina17@unipa.it}

The scientific literature has considered the role of perioral forces in orthodontic treatment planning in children as well as in adults. Although contradictory results have been reported, several authors have demonstrated the relationship between lip and tongue pressure related to Angle classification, occlusion characteristics and oral habits. The aim of this study was to investigate any changes on perioral muscles strength (i.e. tongue, lip muscles and masseter muscles) in children aged 6-11 years with and without orthodontic treatment. Methods: Sixty-eight children, 36 males and 32 females, were recruited for the study and assigned to one of the following two groups: 1) a group composed by children with orthodontics at the moment of the enrollment and children who had received orthodontic treatment prior to the enrollment (OG); 2) a group formed

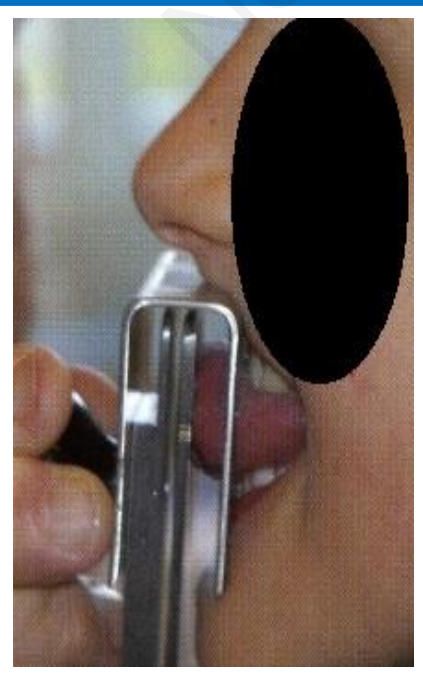

Fig. 11 Procedure for measuring the tongue extension force. by children without orthodontics (NO-OG). All participants completed the protocol assessment that included: a dental occlusion-class evaluation, a swallowing function test with the Payne technique and a measurement of perioral forces [tongue extension force (Figure 11), lip pressure, masseter contraction force] using the Myometer 160 (MFT-Products; Matzendorf, Solothurn, Switzerland). Mean values and standard deviations were calculated using Statistica Software 12 (StatSoft ${ }^{\circledR}$, TIBCO ${ }^{\circledR}$ Software Inc, Palo Alto, CA, USA). Differences between groups were analysed using a t-test. The $\mathrm{p}$-value was set at $\mathrm{p}<0.05$. Results: The perioral forces measurement showed a significant difference $(\mathrm{p}<0.05)$ between OG and NO-OG only for the tongue extension force. In particular, NO-OG indicated values out of the normal range compared to OG, though OG was at the minimum value of the normal range. No significant difference $(p>0.05)$ was found for lip pressure and masseter contraction force between groups. However, the OG showed values of masseter contraction (both right and left) within the physiological range while, the OTG showed lower and dysfunctional strength levels of both masseter muscles. Our results suggest that in children with orthodontic treatment may induce to facial muscular strength levels closer to the normal range. However, orthodontic treatment also requires orofacial myofunctional therapy.

1. Messina G. The Tongue, Mandible, Hyoid System. Eur J Transl Myol 2017;27:6363.

2 Lambrechts H, De Baets E, Fieuws S, Willems G. Lip and tongue pressure in orthodontic patients. Eur J Orthod 2010;32:466-71.

3. Messina $G$, Martines $F$, Thomas E, et al. Treatment of chronic pain associated with bruxism through Myofunctional therapy. Eur J Transl Myol 2017;27:6759.

$* * * * *$

Home based Functional Electrical Stimulation for Denervated Degenerating Muscle: Training parameters optimized for denervation time and age

\section{Christian Hofer 1, Helmut Kern 2, Stefan Löfler 3,} Winfried Mayr 4

1 Ludwig Boltzmann Institute of Electrical Stimulation and Physical Rehabilitation, Wilhelminenspital Wien, Austria; 2 Physiko- und Rheumatherapie, St. Poelten, Austria; 3 Center of Medical Physics and Biomedical Engineering, Medical University of Vienna, Austria

\section{E-mail: christian.hofer@rehabilitationresearch.eu}

The stimulation parameters applied for eliciting muscle contractions are depending on physiological conditions of the muscle. Of particular importance for electrical stimulation is whether the connection between the muscle and the nerve is preserved or the muscle is denervated due to Spinal Cord Injury or peripheral nerve lesion. In the latter cases the denervated muscle becomes unexcitable with commercial electrical stimulators and undergoes ultrastructural disorganization within a few months, while severe atrophy with nuclear clumping and fibro-fatty degeneration appears later on within 3 and 6 


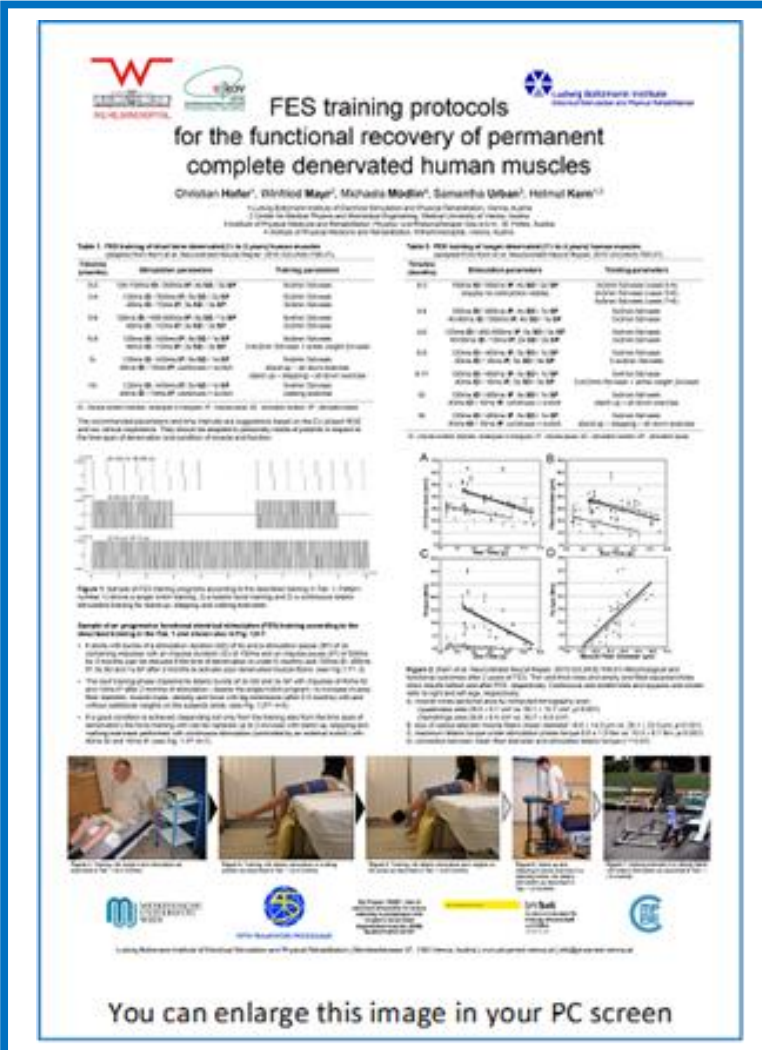

Fig. 12 Training parameters optimized for denervation time and age.

years. ${ }^{1-3}$ This changes are essential for developing stimulation protocols since functional activation of denervated muscles requires electrical stimulation with long impulse duration in the range of $20-150$ (up to 300) ms (Figure 12). Moreover, contrary to innervated muscle where the nerve distributes the action potential in the denervated muscle an electrical field distribution capable of depolarizing the fibers in almost every part of the muscle has to be achieved. Therefore, to counteract the deterioration of the denervated muscle a therapy concept for home-based electrical stimulation was developed. To carry out the training a stimulator suited to deliver the necessary high-intensity and long duration impulses and new electrodes were designed. ${ }^{4,5}$ Specific clinical assessments to monitor the condition of the patient's muscles and guidelines for training were developed at the Wilhelminenspital Wien, Austria. ${ }^{4}$ The novel therapy concept (Figure 13), together with the newly designed devices, was evaluated in the RISE clinical study. After completing the 2-year home-based therapy program the subjects showed a significant increase of muscle mass and of myofiber size, improvements of the ultrastructural organization and recovery of tetanic contractility with significant increase in developed muscle force output during electrical stimulation. ${ }^{5}$ EC approved products (Stimulette den2x) and purpose constructed large safety electrodes are now commercially available (Dr. Schuhfried Medizintechnik Company, Vienna, Austria). ${ }^{6}$

1. Albertin G, Hofer Ch, Zampieri S, et al. In complete SCI patients, long-term Functional Electrical Stimulation of permanent denervated muscles increases epidermis thickness. Neurol Res 2018;40:277-82. doi: 10.1080/01616412.2018.1436877. Epub 2018 Feb 15.

2. Carraro U, Boncompagni S, Gobbo V, et al. Persistent Muscle Fiber Regeneration in Long Term Denervation. Past, Present, Future. Eur J Transl Myol 2015;25(2):4832. doi: 10.4081/ejtm.2015.4832. eCollection 2015 Mar 11. Review.

3. Carraro U, Kern H. Severely Atrophic Human Muscle Fibers with Nuclear Misplacement Survive Many Years of Permanent Denervation. Eur J Transl Myol 2016 Jun 13;26(2):5894. doi: 10.4081/ejtm.2016.5894.

4. Hofer C, Mayr W, Stöhr H, et al. A stimulator for functional activation of denervated muscles. Artif Organs 2002;26:276-9.

5. Kern H, Hofer C, Mayr W, Carraro U. European Project RISE: Partners, protocols, demography. Eur J Transl Myol 2009;19:211-6.

6. Available at http://schuhfriedmed.at/stimulette-en/stimu lette-den $2 x$ -

$* * * * *$

\section{Quantitative light morphometry of elastic fibers and} vessels in skin of DDM SCI persons

Barbara Ravara 1,2,3, Andrea Porzionato 1, Ugo Carraro 2,3, Helmut Kern 4,5, Giovanna Albertin 1

1 Department of Neuroscience, Section of Human Anatomy, University of Padova, Italy; 2

Interdepartmental Research Center of Myology,

Department of Biomedical Science, University of Padova, Italy; 3 A\&C M-C Foundation for

Translational Myology, Padova, Italy; 4 Physiko- und Rheumatherapie, St. Poelten, Austria

\section{Email: giovanna.albertin@unipd.it}

Daily skin care may increase skin regeneration, elasticity, smoothness, and thus delay skin complications, while degradation of skin primary structural constituents, i.e., elastin and fibrous collagen, significantly occurs in old person and may occur in early aging, mimicking the effects of chronic sun exposure. Changes of the micro vessels are alsoan accompaining fenomenon. Literature provides evidence that cell proliferation and migration are positive responses to endogenous electrical currents or external electrical stimulation used to promote these biological processes for the treatment of chronic wounds. Our aims are to evaluate elastic and fibrous collagen fibers, capillaries and lymphatic vessels on skin biopsies harvested from DDM spinal cord injury patients before and after two years of h-bFES. This information may correlate or not with the adaptations recently described on epidermal thickness, ${ }^{1,2}$ and epidermal-dermal morphology that strongly support effectiveness of electrical stimulation in DDM SCI persons. ${ }^{3-4}$ Preliminary results will be reported on skin sections, stained by Verhoff-Van Gieson method. ${ }^{5}$ Furthermore, by immunostaining, blood microvasculature by the CD $31,{ }^{6}$ and lymphatic vessels by LYVE-1 antibodies will be analyzed. Normal skin biopsies harvested during minimally invasive surgery will be stained to follow-up skin changes with age.

1. Albertin G, Hofer C, Zampieri S, et al. In complete SCI patients, long-term functional electrical stimulation of 


\section{Sp PMD Collection of the Abstracts}

Eur J Transl Myol 29 (1):46-78 2019

permanent denervated muscles increases epidermis thickness. Neurol Res 2018;40:277-282. doi: 10.1080/01616412.2018.1436877. Epub 2018 Feb 15.

2. Albertin G, Kern H, Hofer C, et al. Two years of Functional Electrical Stimulation by large surface electrodes for denervated muscles improve skin epidermis in SCI. Eur J Transl Myol. 2018;28(1):7373. doi: 10.4081/ejtm.2018.7373. eCollection 2018 Jan 12.

3. Kern H, Carraro U, Adami N, et al. Home-based functional electrical stimulation rescues permanently denervated muscles in paraplegic patients with complete lower motor neuron lesion. Neurorehabil Neural Repair. 2010;24:709721. DOI:10.1177/1545968310366129. Epub 2010 May 11.

4. Kern H, Carraro U. Home-based functional electrical stimulation ( $h$-b FES) for long- term denervated human muscle: history, basics, results and perspectives of the Vienna rehabilitation strategy. Eur J Transl Myol 2014;24:27-40. DOI: 10.4081/ejtm.2014.3296 eCollection 2014 Mar 31.

5. Diekmann J, Alili L, Scholz, Oet al. A Three-dimensional skin equivalent reflecting some aspects of in vivo aged skin. Exp Dermatol 2016;25:56-61. doi: 10.1111/exd.12866. Epub 2015 Nov 23.

6. Bentov I, Reed MJ. The effect of aging on the cutaneous microvasculature. Microvasc Res 2015;100:25-31. doi: 10.1016/j.mvr.2015.04.004. Epub 2015 Apr 24. Review.

$$
* * * * *
$$

\section{Analysis of EMG signals from the triceps surae muscle in Achilles tendon tests by stroke patients in tSCS treatment}

Thordur Helgason, 1,2 Halldor Karason 1, Vilborg Gudmundsdottir2, Gigja Magnusdottir 2, Belinda

Chenery 3, Gudbjorg Ludwigsdottir 2

1 Reykjavik University, Iceland; 2 LandspitaliUniversity Hospital, Reykjavik, Iceland; 3. University of Iceland, Reykjavik, Iceland

Email: thordur@landspitali.is

****************************************************

\section{Acknowledgments and Funding}

This typescript is sponsored by the A\&C M-C Foundation for Translational Myology, Padova, Italy.

\section{Conflict of Interest}

The author declare to have none conflicts of interests.

\section{Ethical Publication Statement}

Author confirms that he have read the Journal's position on issues involved in ethical publication and affirms that this report is consistent with those guidelines.

\section{Corresponding Author}

Ugo Carraro, Department of Biomedical Sciences, University of Padova, Italy.

E-mail: ugo.carraro@unipd.it

Received for publication: March 08, 2019

Accepted for publication: March 11, 2019 
INDEX

\begin{tabular}{|c|c|c|c|c|c|}
\hline Alaibac M & 69 & Harridge SDR & 53 & Pratt EE & 52 \\
\hline Albertin G & $48,69,71,76$ & Hatakeyama S & 51 & Protasi F & 61,69 \\
\hline Albracht K & 64 & Helgason $\mathrm{T}$ & 77 & Raffaello A & 55 \\
\hline Altmann C & 59 & Helm M & 64 & Raiteri BJ & 66 \\
\hline Anderson L & 52 & Hockerman GH & 52 & Rambaldo A & 69 \\
\hline Angeli CA & 46 & Hofer Ch & $48,60,69,70,75$ & Rantz E & 52 \\
\hline Angelini C & 56 & Hong W & 53 & Ravara B & $8,69,70,71,73,76$ \\
\hline Argenton F & 55 & Iovane A & 75 & Reggiani C & 55,57 \\
\hline Armani A & 54 & Jaka O & 53 & Rinaldi C & 54 \\
\hline Armstrong C & 57 & Jarvis JC & $48,59,72$ & Risato G & 55,74 \\
\hline Arnold D & 58 & Kabas F & 68 & Ritzmann R & 64 \\
\hline Aszmann OC & 60 & Karason $\mathrm{H}$ & 77 & Rizzuto R & 55 \\
\hline Basso M & 54 & Kast C & 60 & Ruck A & 58 \\
\hline Becker L & 53 & Kern H & $48,67,69,70,71,75,76$ & Sacchetto R & 55 \\
\hline Benati M & 51 & Kirsch A & 59,60 & Sajer $\mathrm{S}$ & 68 \\
\hline Bertolucci C & 55 & Kruger M & 54 & Salyer A & 52 \\
\hline Bianco A & 75 & Lakicevic N & 75 & Sambataro F & 54 \\
\hline Blaauw B & 54,54 & Larsson L & 57 & Sandonà D & 55,74 \\
\hline Boncompagni S & 62 & Lavorato M & 61 & Sandri M & 51,54 \\
\hline Brogi M & 66 & Lieberman AP & 54 & Sarabon Nejc & 68 \\
\hline Butera G & 55 & Loefler S & $48,67,75$ & Sartorelli V & 51 \\
\hline Canato M & 55,57 & Ludwigsdottir G & 77 & Sartori R & 51 \\
\hline Caretti G & 51 & Maggio M & 66 & Sauro Guardiero G & 70,73 \\
\hline Carotti M & 55,74 & Magnusdottir G & 77 & Scano M & 55,74 \\
\hline \multirow[t]{2}{*}{ Carraro U } & $6,48,49,52,67$ & Majcher D & 59 & Scaramuzzino C & 54 \\
\hline & $9,70,71,73,76$ & Mastryukova V & 59 & Schönfelder M & 53,53 \\
\hline Cerrel-Bazo H & 47,48 & Marchioretti C & 54 & Scoppa F & 75 \\
\hline Cesare N & 64 & Marcucci L & 57 & Sedliak M & 67 \\
\hline Chenery B & 77 & Mastryukova V & 58,59 & Segatto M & 51 \\
\hline Chivet M & 54 & Mayr W & $58,59,60,75$ & Shipley J & 53 \\
\hline Collie-Duguid E & 53 & Megighian A & 54 & Sinha S & 63 \\
\hline Costelli P & 51 & Merigliano S & 51 & Sinha U & 63 \\
\hline Cvecka J & 67 & Messina $\mathrm{G}$ & 75 & Soardi M & 55,74 \\
\hline D'Antonio M & 54 & Missaglia S & 56 & Solagna $F$ & 54 \\
\hline Dae Ko K & 51 & Missiaglia E & 53 & Sorarù G & 54 \\
\hline Davie JK & 52 & Möller MC & 59 & Sperti C & 51 \\
\hline De Angelis MH & 53 & Monti E & 64,66 & Spörri J & 66 \\
\hline De Caro R & 69 & Mora M & 56 & Staudle B & 64 \\
\hline De Mello V & 53 & Moresi V & 50 & Sun MAC & 53 \\
\hline Dyar KA & 54 & Mothes O & 58 & Tagliaferri S & 66 \\
\hline Eickhoff S & 48 & Musarò A & 68 & Tavian D & 56 \\
\hline Feiner M & 59 & Narici MV & 64,66 & Thielker J & 58,59 \\
\hline Fenizia C & 51 & Nath S & 54 & Ticinesi A & 66 \\
\hline Figeac N & 53 & Nezdad FY & 53 & Tirpakova V & 67 \\
\hline Filippakopoulos P & 51 & Nogara L & 54 & Uhlenhaut $\mathrm{H}$ & 54 \\
\hline Fisher A & 72 & Palma A & 75 & Vargas Luna JL & 60 \\
\hline Fittipaldi R & 51 & Parodi S & 54 & Vargesson $\mathrm{N}$ & 53 \\
\hline Fitze DP & 66 & Pavan P & 64 & Vecellio Reane D & 55 \\
\hline Franchi MV & 66 & Pegoraro E & 54 & Verbrugge $\mathrm{S}$ & 53 \\
\hline Franzini-Armstrong $\mathrm{C}$ & 61 & Pennisi EM & 56 & Viggars MR & 72 \\
\hline Freyler K & 64 & Pennuto M & 54 & Vissing K & 54 \\
\hline Garcia-Munoz A & 53 & Perkins JD & 60 & Volk GF & 58,59 \\
\hline Gerstenberger C & 59,60 & Petrigna L & 75 & Wackerhage $\mathrm{H}$ & 53,53 \\
\hline Giustino V & 75 & Pierobon ES & 51 & Waldvogel, J & 64 \\
\hline Gollhofer A & 64 & Pin F & 51 & WangWH & 52 \\
\hline Gomez D & 53 & Piol D & 54 & Weilbaecher R & 52 \\
\hline Gudmundsdottir V & 77 & Pirazzini M & 54 & Whitmore C & 52 \\
\hline Gugatschka M & 59,60 & Pirino A & 75 & Zammit P & 53 \\
\hline Guidolin D & 69 & Pobbati AV & 53 & Zampieri S & $48,69,70,73$ \\
\hline Guntinas-Lichius O & 58,59 & Polanco JM & 54 & Zanchettin G & 51 \\
\hline Hahn D & 66 & Pond AL & $48,52,69$ & Zare $\mathrm{H}$ & 51 \\
\hline Hamar D & 68 & Porzionato A & $69,71,76$ & Zuccaro E & 54 \\
\hline
\end{tabular}

